\title{
Modeling of electron devices based on two-dimensional materials
}

\author{
E. G. Marin, M. Perucchini, D. Marian, G. Iannaccone, and G. Fiori
}

\begin{abstract}
The advent of graphene and related two-dimensional (2D) materials has attracted the interest of the electron device research community in the last fourteen years. The possibility to boost transistor performance and the prospects to build novel device concepts with $2 \mathrm{D}$ materials and their heterostructures has awakened a strong experimental interest that requires continuous support from modeling. In this paper we review the state of the art in simulation of electron devices based on two-dimensional materials. We outline the main methods to model the electronic bandstructure and to study electron transport, classifying them in terms of accuracy and computational cost. We briefly discuss how they can be combined in a multiscale approach to provide quantitative understanding of the mechanisms determining the operation of electron devices and we examine the application of these methods to the different families of 2D materials. Finally we shortly analyze the main open challenges of modeling 2D-based electron devices.
\end{abstract}

\section{Index Terms}

two-dimensional materials, simulation, modeling, multiscale, density functional theory, GW, Monte Carlo, tightbinding, Wannier functions, k·p, non equilibrium Green functions, conditional wavefunction, drift-diffusion, compact models

\section{INTRODUCTION}

The two-dimensional (2D) materials world has experimented an unstoppable expansion since 2004, when 2D sheets of carbon atoms, namely graphene, were for the first time prepared and subjected to measurement of charge transport under electric field effect [1]. Nowadays several tens of 2D materials have been synthesized [2], and hundreds of alternatives to increase the list have been theoretically proposed [3]-[5]. The 2D family already includes transition metal dichalcogenides (TMDs), the group IV X-enes, i.e. silicene, germanene and stanene, group III- and group IV-monochalcogenides, phosphorene and group-V layered materials, hexagonal-BN and layered oxides, etc., showing very diverse crystal structures (Figure 1-left). But the possibilities of the two-dimensional world are not restricted simply to their constituents. A plethora of combinations are possible (Figure 1-right), with structures of diverse dimensionality built from these 2D blocks forming new artificial materials [6]. Possibilities range from cutting them into quasi-1D nanoribbons, to stacking them in 3D van-der-Waals heterostructures [7]. Heterostructures can also be fabricated laterally from different phases of the same material [8] or with different number of stacked layers [9] resulting in interfaces with different dimensionality. The creation of random networks of nanotubes [10] or flakes [11] and their use in flexible and wearable electronics are a next step in the adventure [12].

Very intriguing physics is emerging in the world of 2D materials, including superconductivity, topological properties, spin transport and magnetism, phase transitions, or charge density waves and applications in photonics and valleytronics are ventured [13], [14]. Even if very auspicious progresses might be expected in these fields, one should not forget that electronics represent a natural spot for 2D materials [15], [16]. At the heart of field-effect transistors is the control of the potential energy of the electron in the channel, and in 2D-material channels this control might be optimal. A quantitative statement of that in a thin body FET is the scaling length $\lambda=\sqrt{t_{\mathrm{s}} t_{\mathrm{ox}}\left(\epsilon_{\mathrm{s}} / \epsilon_{\mathrm{ox}}\right)}$ which is a measure of the lengthscale of variation of the electrostatic potential, where $t_{\mathrm{s}}$ and $\epsilon_{\mathrm{s}}$ are thickness and the dielectric permittivity of the body, respectively, while $t_{\mathrm{ox}}$ and $\epsilon_{\mathrm{ox}}$ and the thickness and the dielectric permittivity of the gate oxide, respectively [17]. The smaller the

All authors are with Dipartimento di Ingegneria dell'Informazione, Università di Pisa, Pisa, 56122, Italy. The authors gratefully acknowledge the support from the European Commission Graphene Flagship Core 1 (grant agreement No. 696656). Funding from the European Research Council under the European Union Horizon 2020 research and innovation programme (grant agreement No. 770047) are gratefully acknowledged. gfiori@mercurio.iet.unipi.it 


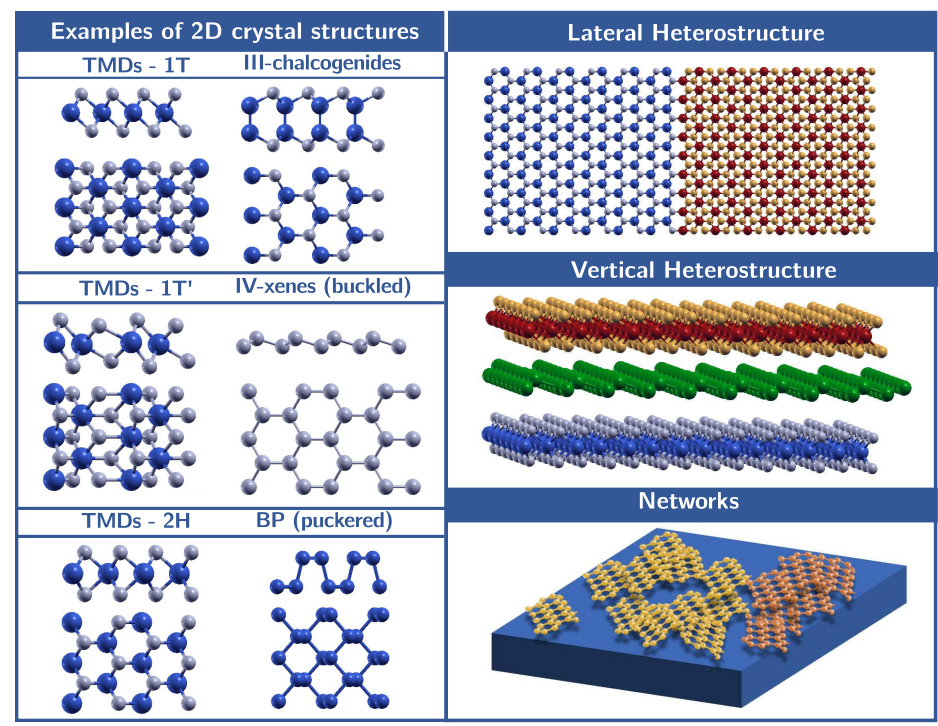

Fig. 1. Left panel: Lateral and top views of the crystal structures present in some 2D materials, e.g. TMDs (left) III-chalcogenides, group IV and black phosphorous (right). Right panel: Structures of diverse dimensionality built from these 2D blocks, including 2D lateral heterostructures (top), 3D vertical or van-der-Waals heterostructures (middle), and networks (bottom).

scaling length, the better the control of the gate over the channel. In this regard, the monoatomic thickness of $2 \mathrm{D}$ materials guarantees the minimum $t_{\mathrm{s}}$ achievable. The other factor determining electronics is how fast carriers can move in the device. Two of the main scattering mechanisms, in particular in FETs, are the surface roughness and the ionized impurities. In both cases 2D materials offer distinct advantages. Regarding surface roughness, 2D materials are free from surface dangling bonds and thus surface roughness is avoided [18]. As for ionized impurities, the fact that most of electric field lines in a 2D material are outside the material itself can be used to dump, by the mediation of the surrounding dielectric, the impact of these impurities [19]. The role of phonons at its impact on the mobility is more controversial [20]. In this context, the application of 2D materials to electronics and to new devices are expected to be in the limelight of the electronics research community in the short- and mid-future.

In spite of this promising potential for electronics and the great advances in the last decade, the experimental techniques in 2D materials are not mature (although big progress in synthesis of wafer-scale graphene [21] and TMDs [22] have been made) and the optimization of an electronic device from every new theoretical alternative becomes prohibitive. It is therefore essential to classify and select, in the increasing constellation of materials, those which might deserve more experimental attention for electronics. Simulation and modeling are, in this context, indispensable tools to perform timely and low-cost exploration, pointing out early the most auspicious paths for experimental investigation. In this review we discuss the main simulation methods to investigate $2 \mathrm{D}$ materials electronic properties as well as to determine the performance of 2D-materialsbased electronic devices. We will pay special attention to how the different methods can be hierarchically ranked in terms of accuracy and computational cost, and how they can be combined in multiscale approaches to provide the most convincing trade-offs between these two aspects. The rest of the paper is structured as follows. In Section II-A we will briefly discuss the main methods for the calculation of the electronic bandstructure of 2D materials and review the main results. In Section II-B, we focus on the device level and simulation approaches. Section III is dedicated to the state of the art in simulation of several families of 2D materials, while in Section IV we point out the main modeling challenges that remain open. Finally we draw our conclusion.

\section{Modeling: THE MUlTiscAle PICTURE}

The complexity of current 2D nanoelectronics requires a versatile modeling approach, able to capture the physical details of the materials and at the same time to provide a high-level description of device operation. To this purpose, there exist a plethora of methods in the simulation community that, depending on the aim of 
study, can be coarsely classified in two categories: materials modeling and device modeling. In each category, these methods can be ranked according to their accuracy and computational demand.

For a proper study of 2D-materials-based electronic devices, these descriptions must be combined in a flexible approach where more accurate results are used at higher abstraction levels, and materials properties feed device modeling. This is the purpose of the multiscale approximation that has become a standard and a increasingly adopted approach in the 2D materials community [23]. The multiscale approach connects the various levels of physical abstraction through well-defined interfaces for exchange of information providing a complete depiction of the device behavior. In the following we will briefly review the main methods employed in the modeling of 2D materials and 2D devices and we will discuss how they are connected.

\section{A. Materials modeling}

All the physics that takes place in a device is determined by the constituting materials, specifically by the precise arrangement of the atoms and their interactions. For an electron device, the main information on the materials is embedded in the electronic bandstructure, i.e. the energetic representation of the allowed electronic states, that provides most of the electronic variables of interest, e.g band-gap, electron affinities, workfunction, density of states, effective masses, etc.

The exact calculation of the electronic bandstructure of a material requires to solve the interacting manyparticle Schrödinger equation, which is unfortunately too complicated to be solved except for extremely simple cases. However, there is a complete hierarchy of methods, based on the Born-Oppenheimer approximation for the nuclei motion, to approach its solution (Figure 2).

At the base of this hierarchy there are ab-initio or first principle methods, including Quantum Monte Carlo, Density Functional Theory and GW approximation. Atomistic models constitute the next level of approximation: they are quantum mechanical in form, but require inputs from ab initio, like Maximally Localized Wannier Functions, and/or from empirical results like the Tight-Binding approximation. A subsequent level is comprised by methods focused at the energies surrounding the bandgap like $\mathrm{k} \cdot \mathrm{p}$. The rank in the material modeling ends up with analytical approximations of the electronic band-structure. In the following we will briefly review these methods and their application to the investigation of $2 \mathrm{D}$ materials.

Quantum Monte Carlo (QMC) The essence of this method relies on the evaluation of a multi-dimensional integral by statistically sampling the integrand and averaging the sampled values. Amongst the different QMC methods, the most popular one for the calculation of the electronic bandstructure are the variational Monte Carlo (VMC) and the diffusion Monte Carlo (DMC) [24]. The former is based on a mix of the variational principle and Monte Carlo estimation of integrals; the latter, more sophisticated and accurate, is based on the projector approach. Both methods rely on a wavefunction ansatz on which the reliability, efficiency and accuracy of the method drastically depend. Both perform calculations of the average total energy of the system $E=\langle\Psi|\hat{\mathcal{H}}| \Psi\rangle /\langle\Psi \mid \Psi\rangle$. Nowadays the QMC techniques in the 2D materials framework are mainly used as a benchmark for testing Density Functional Theory results. Indeed, as they are free of approximations (except for their dependence on the initial trial wave function) these techniques are superior for studying layered systems where van-der-Waals interactions play a crucial role [25], [26], but their extremely high computational cost limits their use.

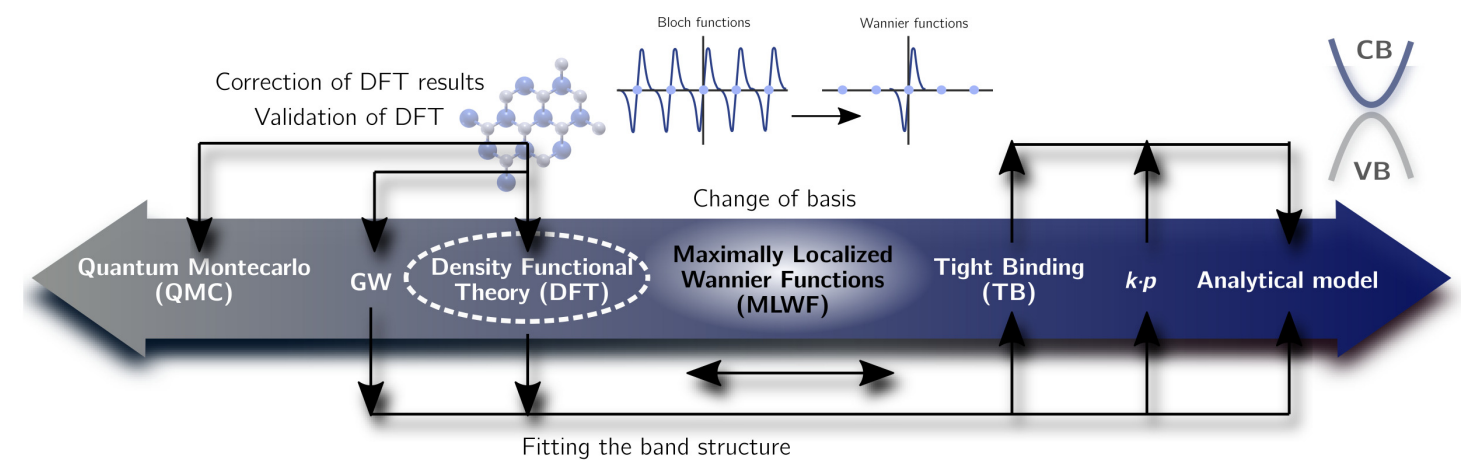

Fig. 2. Scheme of the different levels of approximation for the modeling of materials electronic properties hierarchically ranked according to their accuracy and computational demand. Interfaces for the exchange of information between different methods are defined. 
Density Functional Theory (DFT) is the most widely used method to determine the electronic properties of solid-state systems. In spite of well-known inaccuracy in determining semiconductors bandgap (Figure 3), it is the touchstone for most of bandstructure calculations. The Kohn and Hohenberg theorems [27] establish the mathematical foundation of DFT. The first theorem proves a one-to-one relation between the external potential and the ground charge density for a system with a given number of particles. The second theorem derives that the ground state energy is a functional of the ground charge density and can be determined by using the variational principle. These two theorems are translated into practice by the Kohn and Sham approach [28] where the many-electron interacting problem is substituted by a many-electron non-interacting problem with identical ground state charge density.

The differences between the interacting and non-interacting solutions are absorbed in the so-called exchangecorrelation functional $V_{\mathrm{xc}}$. If $V_{\mathrm{xc}}$ collects exactly these differences, the Kohn-Sham approach is an exact solution of the many-body interacting stationary Schrödinger equation. However, this is so far not possible and the $V_{\mathrm{xc}}$ must be approximated.

There are different possibilities to approximate $V_{\mathrm{xc}}$, with the most common being the Local Density Approximation (LDA) and the Generalized Gradient Approximation (GGA). Its application to the study of 2D materials forms an extensive literature discussing graphene, transition metal dichalcogenides [29], group IV [30] and group V [31] layered materials, III-V layered compounds [32] and practically any 2D material conceived so far [3].

$\boldsymbol{G W}$ reformulates the many-particle Schrödinger equation, under the Green's function theory, into a set of single-particle-Schrödinger-like equations where exchange and correlation effects are included in the socalled self-energies, $\Sigma=i G W$, that give name to the approximation. Here $G$ is the many-particle system Green's function and $W$ is a dynamically screened Coulomb interaction. The set of equations are solved iteratively and self-consistently usually starting from a tentative solution that is taken from a converged DFT simulation. The GW method goes beyond DFT in considering the electron-electron interactions by means of exact, nonlocal Fock exchange and correlation dynamical screening, overcoming DFT problems in the estimation of the bandgap of semiconductors (Figure 3), but at the cost of a higher computational demand. To this purpose, simplified implementations as $G_{0} W_{0}, G W_{0}$ that relax the self-consistency requirements are often employed. GW together with DFT has been used to accurately determine the banstructure and effective masses of a wide collection of 2D materials in [33], what can be later used in device-level abstractions. In particular, for complementary digital logic a sufficiently large bandgap $(>1 \mathrm{eV})$ and symmetric electron $\left(m_{e}\right)$ and holes $\left(m_{h}\right)$ effective masses are desirable. Figure 3 shows $m_{e}$ vs. $m_{h}$ for some 2D materials as obtained from GW along zig-zag and armchair orientations [33].

Tight-binding (TB) method is one of the most used methods in the device community due to its computational efficiency and accuracy. It can be formulated starting with a linear combination of atomic orbitals of Bloch-form localized at each site of the atomic lattice. The tight-binding ansatz wavefunction is written (for one orbital per lattice site) as: $|\Psi\rangle=\sum_{m} e^{i \mathbf{k} \cdot \mathbf{R}_{m}}|m\rangle / \sqrt{N}$, where $|m\rangle$ is the state at site $m, \mathbf{R}_{m}$ is $m$ site lattice vector, and $\mathbf{k}$ is the wavevector. The energy bandstructure can then be obtained by plugging $|\Psi\rangle$ into the Schrodinger equation and projecting it into $\langle\Psi|$ obtaining:

$$
E(\mathbf{k})=\frac{\sum_{n, m} e^{i \mathbf{k} \cdot\left(\mathbf{R}_{m}-\mathbf{R}_{n}\right)}\langle n|\hat{H}| m\rangle}{\sum_{n, m} e^{i \mathbf{k} \cdot\left(\mathbf{R}_{m}-\mathbf{R}_{n}\right)}\langle n \mid m\rangle}
$$

where $\langle n|\hat{H}| m\rangle$ and $\langle n \mid m\rangle$ are used as input parameters obtained empirically or from first principle methods. Several tight-binding models have been developed for 2D materials, making of it the most used approach for bandstructure calculations amongst atomistic methods. It has been employed to study e.g. the bandstructure of TMDs [34], [35], black phosphorous [36], or the effect of the spin-orbit coupling on silicene, germanene and stanene [37], the impact of strain on graphene [38], or the chirality in carbon nanotubes [39]. TB models are able to capture the lower energy bandstructure, but usually have some difficulties in reproducing high energy states with a limited number of nearest neighbors. Its extension to higher unoccupied energies in 2D materials has been treated in [40]. 
Maximally Localized Wannier Functions (MLWFs) method represents the ideal connection between abinitio calculations and tight-binding Hamiltonians. Wannier Functions [41] retains the information of Bloch states, $\psi_{n \mathbf{k}}$, in a single-particle approximation like DFT, such that, the electronic ground state of the system is mapped onto localized Wannier states, $w_{n \mathbf{R}}$.

The mapping of the Bloch states into Wannier functions is not unique, since there is a Gauge freedom [42], with the subsequent implication that from a set of Bloch states one can have different sets of Wannier functions. MLWF represents among these different possibilities the most localized ones. The procedure of seeking the most localized Wannier functions was firstly described for an isolated group of bands [43] and later extended for and entangled group of bands [44] and implemented in the code Wannier90 [45]. The usefulness of passing from extended states to localized states resides on the possibility of deriving a tight-binding-like Hamiltonian from the Wannier functions in a fully automatic fashion, skipping time consuming procedures to obtain optimized set of parameters as in the semi-empirical tight-binding methods, through least-mean square or genetic algorithms. The Wannier Hamiltonian has been successfully adopted in the study of 2D materials to later compute transport properties.

Only to name a few, MLWFs have been used to confirm the presence of edge states in 2D bismuthene alloys [46] calculate transport in arsenene and antimonene 2D FET transistors [47], to determine the Landau levels and the corresponding edge states of several $2 \mathrm{D}$ crystals including graphene, $\mathrm{hBN}, \mathrm{MoS}_{2}$, phosphorene and InSe, [48], to evaluate the performances of 2D FET based on InSe [49], phosphorene and $\mathrm{MoS}_{2}$ devices [50]-[52], to analyze the conductance of functionalized carbon nanotubes [53], to study two dimensional transistor based on heterostructures of $\mathrm{MoS}_{2} 1 \mathrm{~T}$ and $2 \mathrm{H}$ phases [54] and to propose a TFET device based on stanene nanoribbon [55].

$\boldsymbol{k} \cdot \boldsymbol{p}$ method is based on perturbation theory and provides accurate information regarding conduction band minima and/or valence band maxima of the material. A priori information about the main orbitals contributing to these states is needed. The Hamiltonian, $\hat{H}=\hat{H}^{0}+\hat{H}_{\mathbf{k} \cdot \mathbf{p}}+H_{\mathrm{SO}}$, is then built as the combination of the unperturbed Hamiltonian, $\hat{H}^{0}$, including kinetic and external potential energy terms, the $\mathbf{k} \cdot \mathbf{p}$ perturbation $\hat{H}_{\mathrm{k} \cdot \mathrm{p}}$, and a spin-orbit-coupling term, $H_{\mathrm{SO}}$. k.p must be fed by input parameters from ab-initio or tight-binding models, but provides a simple and accurate enough depiction of the bandstructure at low energies. A recent review presents an extensive collection of $k \cdot p$ parameters for transition metal dichalcogenides [56]. The $k \cdot p$ methods has also been employed to model silicene and germanene bandstructures at low energies [37]
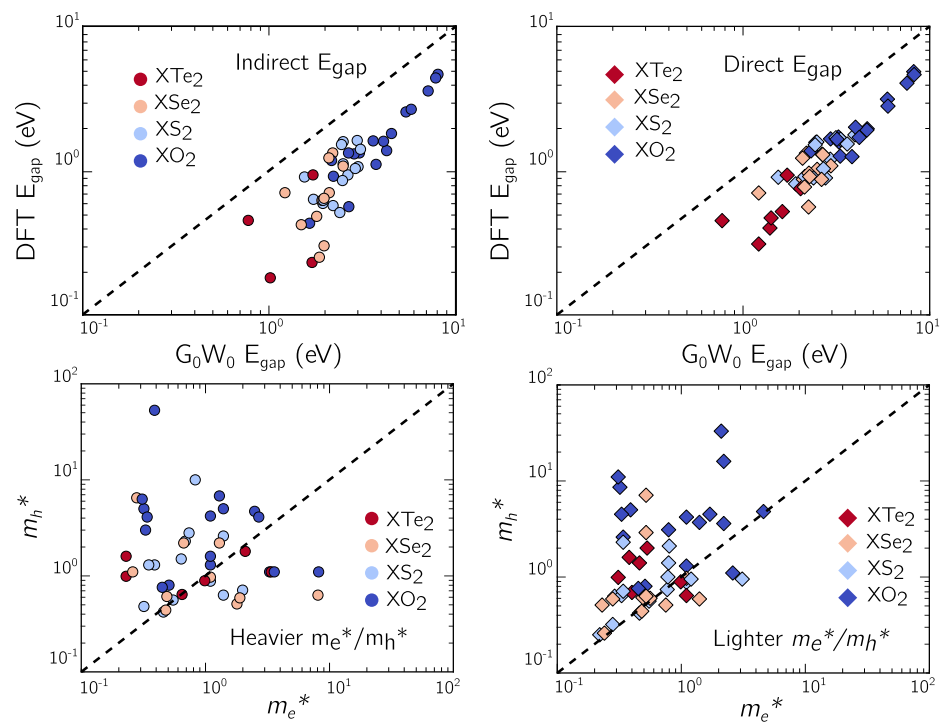

Fig. 3. Top Panel: Indirect and direct bandgaps obtained with DFT ad GW extracted from the Computational Database Repository [33] for different TMDs compounds where $\mathrm{X}$ stands for a transition metal atom of group 4, 6, 10 or Ge and Sn, and the chalcogenide atom is $\mathrm{S}$ (light blue), Se (light red), Te (red), or O (blue). Bottom panel: Electron vs. hole effective masses along the two principal axes of the crystal for the same materials. 


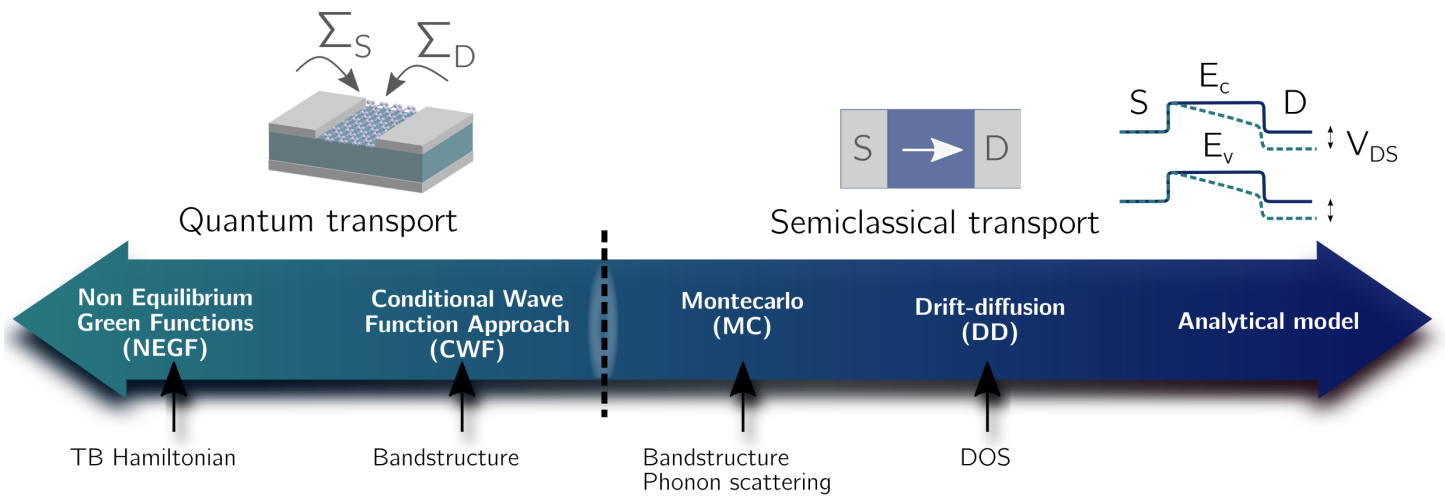

Fig. 4. Different approaches to modeling an electron device hierarchically ranked according to their accuracy and computational demand. The information from the materials bandstructure feeds each method differently.

\section{B. Device modeling}

In order to model the behavior of an electronic device, it is necessary to determine the carrier distribution out of the equilibrium, i.e. when some external voltages are applied at its terminals. Differently from materials modeling, where a quantum description of the electronic bandstructure is mandatory, the length scales involved in device modeling enable to choose between quantum approaches, mostly inspired in the seminal work by R. Landauer [57] and the L. Keldysh formalism [58], and a semi-classical approach based on the Boltzmann Transport Equation (Figure 4). Consequently, the hierarchy of methods includes: i) quantum-based methods like the conditional wave function (CWF) or Non Equilibrium Green Functions (NEGF) and ii) semi-classical descriptions based on the solution of the Boltzmann Transport Equation (BTE), e.g. Monte Carlo transport, Drift-Diffusion (DD). At the bottom of the hierarchy, simplified compact models appear. There is a variety of ways in which the materials description information enters in these methods, from Tight-Binding depictions in NEGF, to definitions of the dispersion relationship at low energies for $\mathrm{CW}$, or the density of states for DD. Nevertheless, for a consistent solution, the study of the device must include the electrical connection between the external potential and the charge carriers by means of the Poisson equation. In the following, we review the aforementioned methods to calculate transport in electronic devices and some of the main studies employing them in 2D based transistors.

\section{Non Equilibrium Green Functions}

(NEGF) formalism solves the open-boundary Schrödinger equation [59]: since electron devices interchange particles and energy with outside, a direct application of the Schrödinger equation (only valid for closed systems) is not possible, and a reformulation of it is needed, i.e $E \psi=H \psi \rightarrow G E=G H+G \Sigma+1$, where $\Sigma$ accounts for the interchange with the outside, and $E, H$ and $G$ are the energy, the Hamiltonian and the Green functions; the latter containing all the information required to study transport in the device and calculated as:

$$
G(E)=\left[E I-H-\Sigma-\Sigma_{p h}\right]^{-1}
$$

were, optionally, $\Sigma_{p h}$ are self-energies accounting for electron-phonon scattering in the device region. NEGF is able to provide important information as the free carrier concentration and the transmission coefficient. When applying the NEGF method to the study of devices, it is important to bear in mind the assumptions it is based upon, namely the single-particle approach and the mean-field approximation. Therefore, this formalism is not suitable in cases of strongly correlated transport or in the 'Coulomb blockade' regime. Nonetheless, it is a central tool in the determination of the transport in 2D-based electronic devices and has been widely employed to determine transport in a variety of 2D-based structures, including carbon nanotubes [60], TMDs transistors [52], [61]-[63] and heterostructures [54], [64], monochalcogenides FETs [49], [50], [65], black phosphorous and other group V layered materials transistors [47], [66], [67], or group IV nanoribbons [55], [68].

Conditional wave function (CWF) approach has been recently developed to study quantum transport in 2D (and 3D) materials. This method arises from Bohmian Mechanics, an alternative version of Quantum Mechanics in which particles have a definite position and their motion is choreographed by the wave function 
[69]-[71]. In particular, the CWF is the wavefunction of a subsystem defined in this theory that serves to propose an alternative solution to the so-called many-body problem. The CWF of each particle evolves under a Schrödinger-like equation where Coulomb interactions and the external voltage are treated exactly, while two unknown potentials, which retain the non-local correlations of the many-body wavefunction, must be approximated [72]. This method has been recently applied to investigate quantum dissipations in two dimensional materials with graphene-like band structure [73].

Monte Carlo (MC) statistical solution of BTE has been the workhorse for the study of transport in semiclassical approaches in traditional semiconductors. It uses statistics to track the trajectory of classical particles in between stochastic scattering events that change the particle's trajectory and that are treated quantummechanically using Fermi's golden rule. MC solution of BTE does not make any assumption about the distribution function and is suitable for high-field scenarios. The statistical solution proves to be a direct description of the dynamics of the carriers in the material. Variations of the MC approach exist depending on the homogeneity and time-dependence of the system, with the ensemble MC method being more appropriate for nanoscale devices. MC has been used to investigate transport in several 2D based electronic devices as in [74]-[78]. In low field scenarios a simpler approach to the BTE is the Momentum Relaxation Time (MRT) approximation. It linearizes the BTE assuming close to equilibrium conditions, i.e small electric fields, uniform transport, i.e the distribution function becomes independent of the transport coordinate, and stationary conditions. The derivation is well documented in the literature [79]. The final result of the approach is an explicit estimation of the scattering rate dependent on a scattering matrix that is determined for every scattering mechanism using Fermi's golden rule and/or ab-initio simulations. These calculations are in any case fed by parameters coming from ab-initio, in particular for phonon scattering. The low-field mobility can later be determined from $\tau$ using the Kubo-Greewood approximation [80], [81], and used to estimate a lowfield current [82]. The MTR approximation is commonly used in conjunction with simplified band-structures based on tight-binding or analytical approximations in the close vicinity of the band minima or maxima. Several works have made use of the MRT to calculate the scattering limited mobility in, e.g. graphene and graphene nanoribbons [82]-[84], $\mathrm{MoS}_{2}$ [19], [85], phosphorene [86], silicene and germanene [87].

Drift-Diffusion (DD) has been one of the most used approximations to model transport in traditional semiconductors and the literature treatment is extensive [88], [89]. It can be derived from BTE under the relaxation time approximation, resulting in independent expressions for the electron/hole current: $J_{\mathrm{n} / \mathrm{p}}=$ $J_{\text {drif }}+J_{\text {diff }}=\rho \mu_{\mathrm{n} / \mathrm{p}} \nabla V \pm q D_{\mathrm{n} / \mathrm{p}}$, where $\rho, \mu$, and $D$ are the charge density, low field mobility and diffusion coefficient, respectively. The latter two can depend on the electric field. DD needs to be solved in conjunction with the continuity equation that might introduce a time-dependent variation of the carrier densities. The bandstructure information for a 2D material is embedded in the calculation of the electron density. DD is specially suitable to compute transport in microscale experimental devices as well as to understand transport in the diffusive regime [90], [91]. A simplified version (while neglecting the diffusive term) is sometimes recalled in the literature invoking linear response theory. A more sophisticated version is the Hydrodynamic model (HD) that also includes heating effects due to high electric fields. The temperature gradient introduces an additional driving force and the current becomes $J_{\mathrm{n} / \mathrm{p}}=J_{\text {drif }}+J_{\text {diff }}+\rho D_{T} \nabla T$, where $D_{T}$ is a thermal diffusivity. HD is a viable alternative to the Monte-Carlo method when dealing with high-field transport and has been used to study the effect of different substrate on graphene at high-field transport [92], providing also a benchmark with experimental results [93].

Compact Models are specially useful to provide concise but rigorous theoretical support to experiments. Depending on the main mechanism determining the current in the $2 \mathrm{D}$ based device, different procedures must be considered. The 'top of the barrier' [94] and the 'gradual channel approximation' [95], [96] are mostly considered to model thermionic FETs, in the ballistic and diffusive regimes respectively, but Boltzmann statistics are many times used to simplify the algebra and find closed expressions [97]. In some cases, Poisson equation is simplified and replaced by a capacitance network [98]. Richardson's approach, particularized for 2D materials have been developed distinguishing the effect of the surface and the edges of flake [99], [100]. It has proven to be useful to explain 2D junctions and diodes. When tunneling phenomena are dominant, the Wentzel-Krammer-Brillouin [101] or Bardeen Transfer Hamiltonian [102] models are more appropriate and 


\begin{tabular}{|c|c|c|c|c|c|}
\hline Methods & $\begin{array}{l}\text { Calc. } \\
\text { time }\end{array}$ & Accuracy & Approximations & Specific problems & $\begin{array}{l}\text { Multiscale } \\
\text { integration }\end{array}$ \\
\hline $\begin{array}{l}\text { Quantum } \\
\text { Monte Carlo }\end{array}$ & $\begin{array}{l}\text { Very } \\
\text { high }\end{array}$ & High & Ir & $\begin{array}{l}\text { Initial trial wavefunction } \\
\text { dependent }\end{array}$ & Low \\
\hline DFT & High & $\begin{array}{l}\text { Medium- } \\
\text { High }\end{array}$ & $\begin{array}{l}\text { Exchange } \\
\text { correlation }\end{array}$ & $\begin{array}{c}\text { Bandgap } \\
\text { underestimation }\end{array}$ & High \\
\hline GW & $\begin{array}{l}\text { Very } \\
\text { high }\end{array}$ & High & $\begin{array}{l}\text { Self-consistency relaxed } \\
\text { for } \mathrm{GW}_{0} / \mathrm{G}_{0} \mathrm{~W}_{0}\end{array}$ & $\begin{array}{l}\text { Low accuracy- } \\
\text { calculation time }\end{array}$ & Medium \\
\hline $\begin{array}{l}\text { ML Wannier } \\
\text { Functions }\end{array}$ & Medium & $\begin{array}{l}\text { Medium- } \\
\text { High }\end{array}$ & $\begin{array}{l}\text { DFT-dependent } \\
\text { results }\end{array}$ & $\begin{array}{l}\text { Initial projection } \\
\text { dependent }\end{array}$ & High \\
\hline $\begin{array}{c}\text { Tight } \\
\text { Binding }\end{array}$ & $\begin{array}{l}\text { Low- } \\
\text { Medium }\end{array}$ & $\begin{array}{l}\text { Low- } \\
\text { High }\end{array}$ & $\begin{array}{l}\text { Orbital set } \\
\text { dependent }\end{array}$ & $\begin{array}{l}\text { Calc. time and accuracy } \\
\text { orbital set dependent }\end{array}$ & High \\
\hline$k$ dot $p$ & Low & Low & $\begin{array}{l}\text { Input parameters } \\
\text { needed }\end{array}$ & $\begin{array}{l}\text { Accurate only for } \\
\text { low-energy bands }\end{array}$ & Medium \\
\hline
\end{tabular}

\begin{tabular}{|c|c|c|c|c|c|}
\hline Methods & $\begin{array}{l}\text { Calc. } \\
\text { time }\end{array}$ & Accuracy & System size & Strengths & Limitations \\
\hline NEGF & High & High & $1-100 \mathrm{~nm}$ & $\begin{array}{c}\text { Versatility and } \\
\text { multiscale integration }\end{array}$ & $\begin{array}{c}\text { Time dependent } \\
\text { calculations, } \\
\text { scattering }\end{array}$ \\
\hline CWF & High & High & $1-100 \mathrm{~nm}$ & $\begin{array}{l}\text { Time dependent and } \\
\text { scattering calculations }\end{array}$ & $\begin{array}{c}\text { Low multiscale } \\
\text { integration }\end{array}$ \\
\hline Monte Carlo & High & High & $0.1-100 \mu \mathrm{m}$ & Versatility & $\begin{array}{c}\text { Not accurate at } \\
\text { quantum level }\end{array}$ \\
\hline $\begin{array}{l}\text { Drift- } \\
\text { Diffusion }\end{array}$ & Medium & Medium & $0.1-100 \mu \mathrm{m}$ & Diffusive regimes & $\begin{array}{l}\text { Not accurate at } \\
\text { quantum level }\end{array}$ \\
\hline $\begin{array}{l}\text { Hydrodynamic } \\
\text { model }\end{array}$ & Medium & Medium & $0.1-100 \mu \mathrm{m}$ & $\begin{array}{l}\text { Captures heating } \\
\text { effects }\end{array}$ & $\begin{array}{c}\text { Not accurate at } \\
\text { quantum level }\end{array}$ \\
\hline $\begin{array}{l}\text { Analytical } \\
\text { models }\end{array}$ & Low & Low & - & Intuitive insight & $\begin{array}{l}\text { Simplified } \\
\text { description }\end{array}$ \\
\hline
\end{tabular}

Fig. 5. Comparison of the material and device methods from different according to their accuracy and computation time. The main approximations/limitations and the strengths of each method are also highlighted.

interesting interlayer tunneling devices been analyzed using these approximations.

In Fig. 5, we show then summarize the main outcomes of the above-mentioned methods, both regarding material and transport models, and specifically how these methods can be classified in terms of accuracy and computing time, highlighting their main strengths and limitations.

\section{Modeling of 2D-MATERIALS-BASED DEVICES}

Here we focus on the main progress made in the modeling of 2D-based electron devices in the last years, organizing the discussion in materials families. We start the discussion, while studying the expected performance of thermionic devices exploiting 2D materials in terms of Power-Delay-Product (PDP), intrinsic delay time $(\tau), I_{\mathrm{ON}}, I_{\mathrm{OFF}}$ ratio and Subthreshold-Swing (SS) as in Fig. 6.

In particular, Figure 6a shows PDP vs. $\tau$ for different $2 \mathrm{D}$ based devices obtained from recent multiscale studies [49], [54], [55]. The simulated devices are defectless transistors, with ideal contact resistance and no scattering in the channel, representing the ultimate performance one may expect, while exploiting such materials (i.e., within the best case scenario). The specifications of the ITRS [103] -recently substituted by the
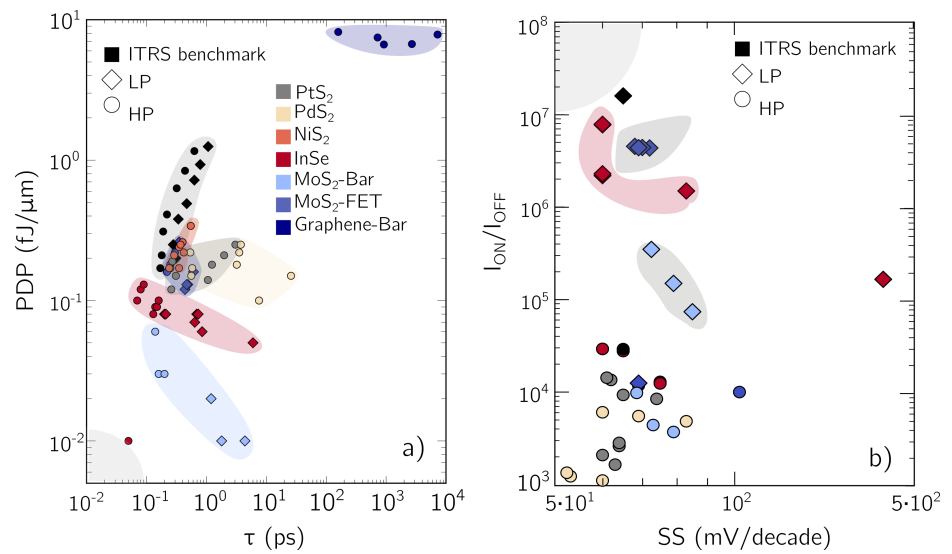

Fig. 6. a) PDP vs. $\tau$ and b) SS vs. ON/OFF ratio for FETs with channels of different 2D materials obtained from multiscale simulations [49], [54], [55]. 
IRDS [104] in its mission of defining a device roadmap- are included for comparison purposes and establish the minimum requirements for the devices to be technologically competitive. Circles and diamonds symbols correspond to high performance and low power applications, that according to the ITRS constraints for static consumption satisfy currents in OFF state $\left(I_{\mathrm{OFF}}\right)$ of $100 \mathrm{nA} \mu \mathrm{m}^{-1}$ and $100 \mathrm{pA} \mu \mathrm{m}^{-1}$, respectively. The preferred corner in Figure 6a is the bottom left, and the different 2D materials families are are shaded in colors. In order to optimize the PDP vs. $\tau$ picture it is necessary to increase the ON state current $\left(I_{\mathrm{ON}}\right)$ keeping low the supply voltage $\left(V_{\mathrm{DD}}\right)$ and the transistor total capacitance $(C)$ because $\tau=C V_{\mathrm{DD}} / I_{\mathrm{ON}}$ and $\mathrm{PDP}=C V_{\mathrm{DD}}^{2}$. This is achieved if the gate has a good electrostratic control on the channel, that can be evaluated through the subthreshold swing (SS). Figure $6 \mathrm{~b}$ shows the $I_{\mathrm{ON}} / I_{\mathrm{OFF}}$ ratio vs. SS. The preferred corner in this picture is the top-left. The $I_{\mathrm{ON}} / I_{\mathrm{OFF}}$ ratio and SS are very affected by the device length and similar lengths ranging from $\sim 5 \mathrm{~nm}$ to $\sim 10 \mathrm{~nm}$ have been considered. It is apparent that most of the structures do comply with the ITRS requirements and would deserve more critical testing to determine their performance in non-ideal conditions.

Once summarized the big picture, we focus now on reviewing the electron device modeling advances in the better known families of $2 \mathrm{D}$ materials.

Graphene is the most investigated 2D crystal. Its study has been covered for almost every physical perspective, ranging from excitons and spin transport to antiferromagnetism in nanoribbons or stacked crystals. There are several excellent reviews focusing on the basic science of graphene [105] up to its application in electronics [106]. The well-know zero bandgap of monolayer graphene jeopardizes its use in FETs and more generally in logic devices, but it has been proposed for RF applications or tunneling transistors [101], [107].

When stacked as in bilayer graphene, the bandstructure becomes sensitive to the vertical electric field, property which has been assessed in both thermionic [108] and Tunnel FETs [109]. Heterostructures combining graphene with other 2D materials have attracted an intense experimental interest, and different multi-scale studies combining ab-initio methods and atomistic transport have been realized [23]. Transport studies based on Monte Carlo [74]-[76] and Drif-Diffusion [90] with the scattering rates and mobility obtained from ab-initio have also been performed [110].

Group-IV 2D-Xenes: silicene, germanene, stanene structures are similar to graphene but slightly buckled (see Fig. 1), due to their larger interatomic distances, which in turn leads to spin-orbit interaction (SOI) [111]. SOI has been investigated from ab-initio and atomistic methods and it has been shown to open small gaps in these 2D crystals [112], [113]. First-principles calculations have shown that the bandstructure can be tuned with a vertical electric field, although the required electric fields are so large that would break any known field oxide in the FETs. [113], [114].

An option explored through ab-initio simulations consists in functionalizing the materials to open larger gaps [115]. Nonetheless, when cut in armchair nanoribbons, a linear dispersion relationship has been predicted from ab-initio and tight-binding models showing a periodic gap dependence on the nanoribbon width [113], [116]. When cut in zig-zag nanoribbons, on the contrary, they show topologically protected states [117], localized at the edges [113], [118], [119]. This would enable ideal conduction through free-of-scattering edges.

On the basis of edge-state transport, a zig-zag stanene nanoribbon transistor based on semi-metallic states has been studied with atomistic models leveraging the possibility of switching the current via scattering modulation [120]. If the nanoribbon is narrow enough, an antiferromagnetic order might prevail with edges of different spin at conduction band minima and valence band maxima, which can be exploited in TFET as demonstrated for stanene by means of multiscale calculations in [55]. Theoretical investigation of FETs based on these materials have also been discussed using ab-initio and atomistic methods [94], [121] as well as Monte Carlo transport simulations [78] and it mobility estimated from MRT [87].

Transition metal dichalchogenides (TMDs) electronic properties have been extensively studied via firstprinciple methods showing a wide range of behaviors: from metallic $\mathrm{NbS}_{2}$ to insulating $\mathrm{HfS}_{2}$ including better known semiconducting $\mathrm{MoS}_{2}, \mathrm{WSe}_{2}, \mathrm{MoSe}_{2}$, just to name a few [122]-[124]. However, the band structure is determined not only by the atomic composition and the metal coordination, but also by layer stacking. TMDs have attracted particular attention for electronics due to their semiconducting bandgap that, in addition, undergoes an indirect-to-direct transition when thickness is reduced from bulk to the monolayer. 
The bandstructure is quite convoluted: eleven orbitals are believed to be relevant in most situations, 6 $p$-orbitals from the chalcogen atoms and $5 d$-orbitals from the metal, which are responsible for spin-orbit coupling [124]. For this reason, single-orbital tight-binding models fail to represent their complexity and models with up to 11 bands are necessary [125], although the simplest alternatives for monolayers based on $k \cdot p$ are available [126]. The heavy electron effective mass in TMDs together with their low in-plane dielectric constant initially placed these materials as good candidates for ultra-short channel FETs [51], [127] and also TFETs [64], but nowadays many more of their properties are being explored and more applications in electronics are found. One of them is the possibility of combining them as building blocks to form lateral or vertical van der Waals heterostructures ( $\mathrm{LH}$ and $\mathrm{VH}$, respectively) with tailor-made characteristics.

The degree of heterogeneity of materials may vary significantly: from quantum-engineered structures involving layers with no atomic species in common, completely different band structures and incommensurate lattices, to band-engineered structures. Interestingly, in the case of $\mathrm{MoS}_{2}$ one can take advantage of the change from the metallic $1 T$ - to the semiconducting crystalline $2 H$-phase to realize a single-material LH [54]. More recently, their application as memristive devices is attracting attention with some theoretical investigation of their use as phase-change materials [128]-[130].

In addition to well-documented TMDs, a new sub-family has recently emerged combining noble transition metals, Pt, Pd, and Ni, with chalcogenides that are so called Noble TMDs [131], [132]. They are predicted to have a strong gap dependence on the number of stacked layers, leading in some cases to a change of electronic phase, from semiconductor to metal. The so-called noble-TMDs are promising contenders to build electronic devices as are expected to have high carrier mobility [133]. The modeling of devices based on these materials is still preliminary [134].

Black Phosphorous (BP) or phosphorene has a honeycomb structure, as graphene and other monolayer materials, but puckered (see Fig. 1) leading to a semiconducting bandgap and a high electrical and thermal anisotropy [135], [136]. Experimental realizations of FETs based on Black Phosphorous have been demonstrated [137] and their performance in the ballistic regime by means of multiscale simulations has been assessed [50], [138], [139]. Its quasi-1D and 3D forms have also been investigated [140]. An alternative allotrope of phosphorene is Blue Phosphorous, with a larger single-layer gap which might be of interest for thermionic devices [141]. Other group-V 2D materials, arsenene, antimonene and bismuthene have also been studied for electron device applications [31], [142]. Arsenene and Antimonene, differently from black phosphorous, present a buckled structure similar to group IV 2D-Xenes. Their mobility has been studied from first principles [143] and their performance in Field Effect Transistors has been studied with a multiscale study combining DFT and NEGF [47]. Bismuthene electronic bandstructure has been investigated from first principles [144] and it is predicted to be a room temperature topological insulator on top of SiC [145]. From the device perspective, bismuthene has received limited attention. However a $2 \mathrm{D}$ crystal compound of $\mathrm{Bi}$, $\mathrm{Bi}_{2} \mathrm{Se}_{3}$ is on the limelight due to its topological properties [46] and its use in Tunnel FET as well as other devices has been discussed [146].

Group III and group IV monochalcogenides. This sub-family of 2D crystals includes combinations of group III elements (e.g Ga and In) or group IV (e.g. Ge, Sn,) with chalcogenides. They are also referred as post-TMDs. In particular, group IV chalcogenides present two puckered atomic planes similar to those of black phosphorus [147], [148]. They have been predicted to have a strong spontaneous ferroelectric polarization by ab-initio calculations, [149] which might be of use to achieve devices with negative quantum capacitance. However, comprehensive theoretical studies of electron transport and of the application of these materials to electron devices are still lacking. Group III chalcogenides, that consist of four atomic planes in a sequence Y-X-X-Y in which Y and X are chalcogenides and group III atoms (see Fig. 1), respectively, have been studied from ab-initio calculations [150], [151]. They present reasonable bandgap for digital logic and intrinsically large mobility. As a consequence, they have been extensively explored as channel materials in FETs by means of multiscale approaches combining ab-initio calculations and quantum transport in the ballistic regime and show compelling performance with respect to industry requirements [49], [50], [65]. In addition a particular crystal phase of InSe has been predicted by ab initio calculations to have a phase transition under strain [152]. 


\section{Challenges AND OPPORTUNitiES}

We finally discuss some of the challenges concerning two-dimensional materials and their application to electron devices that have not been fully addressed so far, and which certainly deserve further investigations from the simulation and modeling perspective (Figure 7).

Contact resistance. Contact resistance is the main limiting factor of the performance of 2D-based devices. It limits the injection of carriers in the device from the external metal leads. For an industrial adoption of the 2D materials technology, values of the contact resistance under $100 \Omega \mu \mathrm{m}$ are required [103] and under $10 \Omega \mu \mathrm{m}$ to be on par with CMOS [16]. The inclusion of degenerate doped regions, as in traditional semiconductors, is desirable but the precise control of the doping is still experimentally challenging. A more novel approach are metallic phases of 2D materials, that are being explored theoretically both from ab-initio and atomistic models [153], [154]. Understanding contact resistance often requires ab-initio modeling of the interface between 2D material and metal and of its transport properties [155], [156], but very often also requires significant support from experiments, in order to gather information about possible interdiffusion or formation of other compounds at the interface, which can significantly alter its transport properties.

Interfaces of different dimensionality. The new perspective of dimensionality introduced with $2 \mathrm{D}$ crystals is pushing towards the exploration of new heterostructures with mixed dimensions and their applications [157]. The problem of contact resistance in 3D-metals-to-2D-materials is a case of interest in electronics. Other mixed interfaces combining 2D crystals and OD, 1D and 3D structures are emerging and can have interesting applications for electron devices (e.g. the patterned graphene contacts have demonstrated better injection due to 1D-edge-to-3D-metal interfaces). In particular, the mixed interfaces are more complex than the ones appearing in traditional heterostructures, as they add to the discontinuity in the bandstructure a steep transition in the density of states as a consequence of the abrupt change of dimensionality. The interacting forces might be van-der-Waals or result from orbitals hybridization, depending on the structure. These interfaces have been studied from ab-initio or semi-classical approaches, but better insight and simpler models on the main principles governing the change of dimensionality will be required.

Surface functionalization In 2D materials, the crystal symmetry is broken at the edges, which play an important role [158]. The way the edges are passivated have an influence on the bandstructure, and several ab-initio studies have looked at their impact on graphene [159], and TMDs [160]. Intentional introduction of defects or 1D molecules in the 2D surface in order to modify the bandstructure through functionalization has been investigated in [161], [162]. Differently from 3D semiconductors, charge transport properties can be engineered by means of functionalized $2 \mathrm{D}$ materials, which can be at the basis of new device concepts, and which might deserve attention from the point of view of modeling.

Transport through vertical or van der Waals heterostructures. A very interesting possibility to be explored with 2D material is the creation of vertical heterostructures [7], [16], [163]. The absence of interlayer covalent bonds and the weak van-der-Waals forces holding the layers together avoid issues related to lattice mismatches although they are not completely free from imperfections [164]. The stacking of different materials phases and the unique intrinsic quasi-electric fields created between them are of strong interest for electronics. In spite of the high experimental interest in these structures and the modeling efforts [165]-[167], there is still a big space for improvement on the knowledge of the principles that govern the electronic transport in vertical heterostructures as well as on the impact that rotational alignment can have on them. A critical aspect is the fact that the transversal energy dispersion relationship of adjacent layers can be totally different, so that the simple physical picture of the one-dimensional band profile can lead to gross errors [168]. For this reason, there is an urgent need for a model that is simpler from the computational point of view than ab-initio, but that includes the relevant physics. One step in this direction has been the introduction of pseudoatomistic Hamiltonians [146], that on the one hand take into account the variations of the energy dispersion relationship and the of the crystalline structure between adjacent layers, but on the other hand are much simpler than atomistic tight-binding Hamiltonians.

High frequency performance and noise. Due to the increasing demand of bandwidth for telecommunications, electron devices are expected to enter deep into the $\mathrm{THz}$ regime, and the impact of 2DMs in this scenario is to be determined. In addition, noise behavior is more and more important both for analog high 


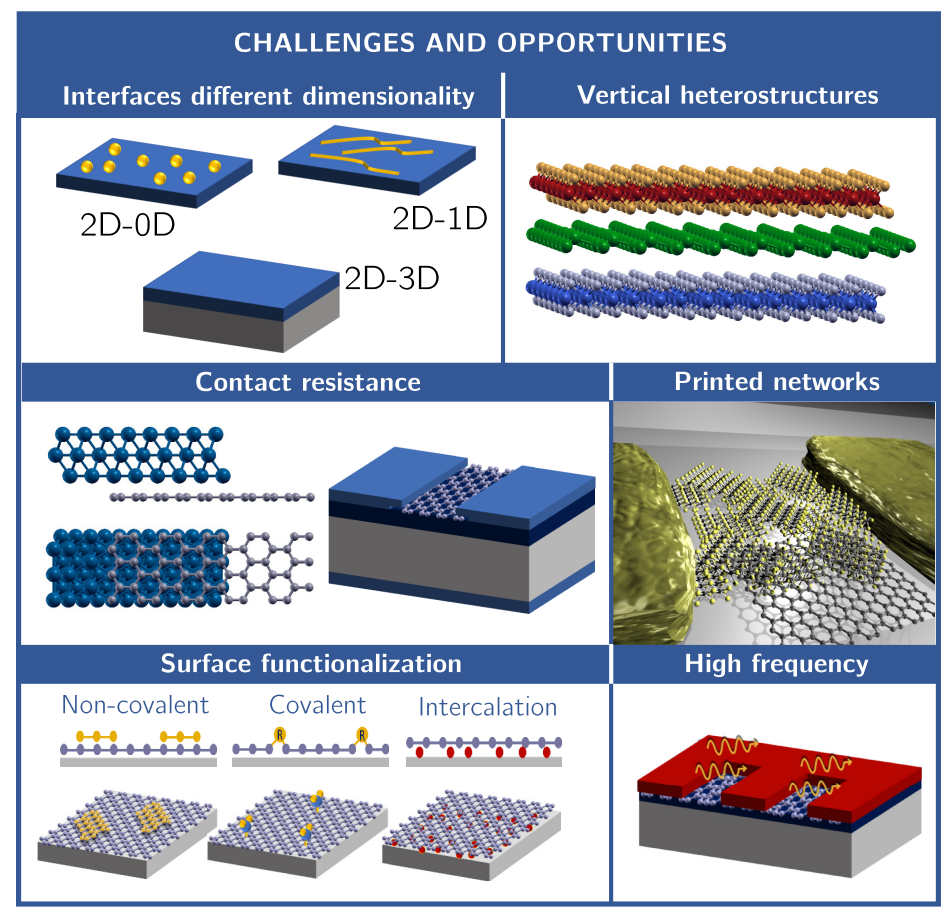

Fig. 7. Challenges and opportunities for the modeling of 2D materials and their application to electron devices.

frequency applications and for low supply voltage. In order to properly model both high frequency behavior and noise, it is often necessary to abandon the quasi-static approximation and to embrace full time-dependent modeling of quantum transport, which is much more demanding from the computational point of view. The topic is particularly challenging also from a purely theoretical standpoint because the separation of devices in quantum regions and reservoirs, typical of the Llandauer-Buttiker approach to quantum transport and noise, does not hold in a simplistic manner.

Printed networks. Flexible devices might be a big technological revolution in electronics [12] and they will be very likely based on printed materials. Indeed, the experimental realization of inkjet printable formulations of 2D materials is progressing rapidly and water-based inks are already available for a range of two dimensional materials including graphene and TMDs [169]. The complexity and randomness of the generated networks requires a multiscale modeling approach. Some theoretical work has been developed in the field of networks of carbon nanotubes and percolation theory, providing even analytical expression in some simplistic scenarios [170]-[172]. The upgrade of this models to 2D materials networks, characterized by a greater interconnection complexity, and the understanding of the mechanisms controlling the current transport in these networks, as well as the devices that would emerge from them, will be an important topic in the simulation and modeling community of 2D materials.

\section{CONCLUSION}

The rise of 2D materials has opened a new phase in the investigation of electronic devices. The exploration of materials and their application to electronics are still at an early stage. However, the novelty in the physics that 2D materials are bringing guarantees promising opportunities for novel device concepts. In order to evaluate these new avenues in electronics, a strong theoretical support to the experimental progress will be needed. In this context, a multiscale approach that combines different levels of accuracy and complexity for materials and device description will be mandatory. However, while the theory seems to keep the pace of experiments in the description of the physical properties of the new synthesized materials, mainly with abinitio methods, the design and study of electronic devices would require increasing efforts to address the best technological options for nanoelectronics. New proposals able to take advantage of the distinct peculiarities of 2D materials have to be introduced, and conceptual advances to address some of the current modeling challenges will be required. Nonetheless, coupled devices/materials modeling for electronics will continued 
to be an essential tool for technology progress, with the multiscale approach very likely playing the role of today's semi-classical TCAD software.

\section{REFERENCES}

[1] K. S. Novoselov, A. K. Geim, S. V. Morozov, D. Jiang, Y. Zhang, S. V. Dubonos, I. V. Grigorieva, and A. A. Firsov, "Electric field effect in atomically thin carbon films," Science, vol. 306, no. 5696, pp. 666-669, 2004. DOI:10.1126/science.1102896

[2] S. Z. Butler, S. M. Hollen, L. Cao, Y. Cui, J. A. Gupta, H. R. Gutiérrez, T. F. Heinz, S. S. Hong, J. Huang, A. F. Ismach et al., "Progress, challenges, and opportunities in two-dimensional materials beyond graphene," ACS nano, vol. 7, no. 4, pp. 2898-2926, 2013. DOI:10.1021/nn400280c

[3] P. Miró, M. Audiffred, and T. Heine, "An atlas of two-dimensional materials," Chem. Soc. Rev., vol. 43, no. 18, pp. 6537-6554, 2014. DOI:10.1039/C4CS00102H

[4] J. T. Paul, A. K. Singh, Z. Dong, H. Zhuang, B. C. Revard, B. Rijal, M. Ashton, A. Linscheid, M. Blonsky, D. Gluhovic, J. Guo, and R. G. Hennig, "Computational methods for 2D materials : discovery , property characterization , and application design," 2017. DOI:10.1088/1361-648X/aa9305

[5] N. Mounet, M. Gibertini, P. Schwaller, D. Campi, A. Merkys, A. Marrazzo, T. Sohier, I. E. Castelli, A. Cepellotti, G. Pizzi et al., "Twodimensional materials from high-throughput computational exfoliation of experimentally known compounds," Nature nanotechnology, p. 1, 2018. DOI:10.1038/s41565-017-0035-5

[6] K. S. Novoselov and A. H. Castro Neto, "Two-dimensional crystals-based heterostructures: Materials with tailored properties," Physica Scripta, no. T146, 2012. DOI:10.1088/0031-8949/2012/T146/014006

[7] A. K. Geim and I. V. Grigorieva, "Van der Waals heterostructures," Nature, vol. 499, no. 7459, pp. 419-425, 2013. DOI:10.1038/nature12385

[8] R. Kappera, D. Voiry, S. E. Yalcin, B. Branch, G. Gupta, A. D. Mohite, and M. Chhowalla, "Phase-engineered low-resistance contacts for ultrathin MoS2transistors," Nature Materials, vol. 13, no. 12, pp. 1128-1134, 2014. DOI:10.1038/nmat4080

[9] X. Duan, C. Wang, J. C. Shaw, R. Cheng, Y. Chen, H. Li, X. Wu, Y. Tang, Q. Zhang, A. Pan et al., "Lateral epitaxial growth of twodimensional layered semiconductor heterojunctions," Nature nanotechnology, vol. 9, no. 12, p. 1024, 2014. DOI:10.1038/nnano.2014.222

[10] S. J. Kang, C. Kocabas, T. Ozel, M. Shim, N. Pimparkar, M. A. Alam, S. V. Rotkin, and J. A. Rogers, "High-performance electronics using dense, perfectly aligned arrays of single-walled carbon nanotubes," Nature Nanotechnology, vol. 2, no. 4, pp. 230-236, 2007. DOI:10.1038/nnano.2007.77

[11] T. Carey, S. Cacovich, G. Divitini, J. Ren, A. Mansouri, J. M. Kim, C. Wang, C. Ducati, R. Sordan, and F. Torrisi, "Fully inkjet-printed two-dimensional material field-effect heterojunctions for wearable and textile electronics," Nature Communications, vol. 8, no. 1, 2017. DOI:10.1038/s41467-017-01210-2

[12] D. Akinwande, N. Petrone, and J. Hone, "Two-dimensional flexible nanoelectronics," Nature Communications, vol. 5, pp. 1-12, 2014. DOI:10.1038/ncomms6678

[13] J. R. Schaibley, H. Yu, G. Clark, P. Rivera, J. S. Ross, K. L. Seyler, W. Yao, and X. Xu, "Valleytronics in 2D materials," Nature Reviews Materials, vol. 1, no. 11, 2016. DOI:10.1038/natrevmats.2016.55

[14] F. Xia, H. Wang, D. Xiao, M. Dubey, and A. Ramasubramaniam, "Two-dimensional material nanophotonics," Nature Photonics, vol. 8, no. 12 , p. 899,2014 . DOI:10.1038/nphoton.2014.271

[15] G. Fiori, F. Bonaccorso, G. Iannaccone, T. Palacios, D. Neumaier, A. Seabaugh, S. K. Banerjee, and L. Colombo, "Electronics based on two-dimensional materials," Nature Nanotechnology, vol. 9, no. 10, pp. 768-779, 2014. DOI:10.1038/nnano.2014.207

[16] G. Iannaccone, F. Bonaccorso, L. Colombo, and G. Fiori, "Quantum engineering of transistors based on 2D materials heterostructures," Nature Nanotechnology, vol. 13, no. 3, pp. 183-191, 2018. DOI:10.1038/s41565-018-0082-6

[17] I. Ferain, C. A. Colinge, and J.-P. Colinge, "Multigate transistors as the future of classical metal-oxide-semiconductor field-effect transistors," Nature, vol. 479, no. 7373, p. 310, 2011. DOI:10.1038/nature10676

[18] M. Chhowalla, D. Jena, and H. Zhang, "Two-dimensional semiconductors for Transistors," Nature Review Materials, vol. 1, 2016. DOI:10.1038/natrevmats2016.52

[19] N. Ma and D. Jena, "Charge scattering and mobility in atomically thin semiconductors," Physical Review X, vol. 4, no. 1, p. $011043,2014$. DOI:10.1103/PhysRevX.4.011043

[20] G. Gaddemane, W. G. Vandenberghe, M. L. Van de Put, S. Chen, S. Tiwari, E. Chen, and M. V. Fischetti, "Theoretical studies of electronic transport in mono-and bi-layer phosphorene: A critical overview," arXiv preprint arXiv:1801.08606, 2018.

[21] Y. Lee, S. Bae, H. Jang, S. Jang, S.-E. Zhu, S. H. Sim, Y. I. Song, B. H. Hong, and J.-H. Ahn, "Wafer-scale synthesis and transfer of graphene films," Nano letters, vol. 10, no. 2, pp. 490-493, 2010. DOI:10.1021/n1903272n

[22] K. Kang, S. Xie, L. Huang, Y. Han, P. Y. Huang, K. F. Mak, C. J. Kim, D. Muller, and J. Park, "High-mobility three-atom-thick semiconducting films with wafer-scale homogeneity," Nature, vol. 520, no. 7549, pp. 656-660, 2015. DOI:10.1038/nature14417

[23] G. Fiori and G. Iannaccone, "Multiscale modeling for graphene-based nanoscale transistors," Proceedings of the IEEE, vol. 101, no. 7, pp. 1653-1669, 2013. DOI:10.1109/JPROC.2013.2259451

[24] W. Foulkes, L. Mitas, R. Needs, and G. Rajagopal, "Quantum monte carlo simulations of solids," Reviews of Modern Physics, vol. 73, no. 1, p. 33, 2001. DOI:10.1103/RevModPhys.73.33

[25] L. Shulenburger, A. D. Baczewski, Z. Zhu, J. Guan, and D. Tomanek, "The nature of the interlayer interaction in bulk and few-layer phosphorus," Nano letters, vol. 15, no. 12, pp. 8170-8175, 2015. DOI:10.1021/acs.nanolett.5b03615

[26] E. Mostaani, N. Drummond, and V. Falko, "Quantum monte carlo calculation of the binding energy of bilayer graphene," Physical review letters, vol. 115, no. 11, p. 115501, 2015. DOI:10.1103/PhysRevLett.115.115501

[27] P. Hohenberg and W. Kohn, "Inhomogeneous electron gas," Physical Review, vol. 136, no. 3B, p. B864, 1964. DOI:10.1103/PhysRev.136.B864

[28] W. Kohn and L. J. Sham, "Self-consistent equations including exchange and correlation effects," Physical Review, vol. 140, no. 4A, 1965. DOI:10.1103/PhysRev.140.A1133

[29] A. Kumar and P. Ahluwalia, "Electronic structure of transition metal dichalcogenides monolayers $1 \mathrm{~h}-\mathrm{mx}_{2}$ from ab-initio theory: new direct band gap semiconductors," The European Physical Journal B, vol. 85, no. 6, p. 186, 2012. DOI:10.1140/epjb/e2012-30070-x 
[30] S. Cahangirov, M. Topsakal, E. Aktürk, H. Şahin, and S. Ciraci, "Two-and one-dimensional honeycomb structures of silicon and germanium," Physical review letters, vol. 102, no. 23, p. 236804, 2009. DOI:10.1103/PhysRevLett.102.236804

[31] M. Pumera and Z. Sofer, "2D Monoelemental Arsenene, Antimonene, and Bismuthene: Beyond Black Phosphorus," Advanced Materials, vol. 29, no. 21, 2017. DOI:10.1002/adma.201605299

[32] H. Sahin, S. Cahangirov, M. Topsakal, E. Bekaroglu, E. Akturk, R. T. Senger, and S. Ciraci, "Monolayer honeycomb structures of group-IV elements and III-V binary compounds: First-principles calculations," Physical Review B - Condensed Matter and Materials Physics, vol. 80, no. 15, pp. 1-12, 2009. DOI:10.1103/PhysRevB.80.155453

[33] F. A. Rasmussen and K. S. Thygesen, "Computational 2d materials database: electronic structure of transition-metal dichalcogenides and oxides," The Journal of Physical Chemistry C, vol. 119, no. 23, pp. 13 169-13 183, 2015.

[34] G.-B. Liu, W.-Y. Shan, Y. Yao, W. Yao, and D. Xiao, "Three-band tight-binding model for monolayers of group-vib transition metal dichalcogenides," Physical Review B, vol. 88, no. 8, p. 085433, 2013. DOI:10.1103/PhysRevB.88.085433

[35] S. Fang, R. K. Defo, S. N. Shirodkar, S. Lieu, G. A. Tritsaris, and E. Kaxiras, "Ab initio tight-binding hamiltonian for transition metal dichalcogenides," Physical Review B, vol. 92, no. 20, p. 205108, 2015. DOI:10.1103/PhysRevB.92.205108

[36] A. N. Rudenko and M. I. Katsnelson, "Quasiparticle band structure and tight-binding model for single-and bilayer black phosphorus," Physical Review B, vol. 89, no. 20, p. 201408, 2014. DOI:10.1103/PhysRevB.89.201408

[37] C. C. Liu, H. Jiang, and Y. Yao, "Low-energy effective Hamiltonian involving spin-orbit coupling in silicene and two-dimensional germanium and tin," Physical Review B - Condensed Matter and Materials Physics, vol. 84, no. 19, pp. 1-11, 2011. DOI:10.1103/PhysRevB.84.195430

[38] V. M. Pereira, A. C. Neto, and N. Peres, "Tight-binding approach to uniaxial strain in graphene," Physical Review B, vol. 80, no. 4, p. 045401, 2009. DOI:10.1103/PhysRevB.80.045401

[39] J. Jiang, R. Saito, G. G. Samsonidze, A. Jorio, S. Chou, G. Dresselhaus, and M. Dresselhaus, "Chirality dependence of exciton effects in single-wall carbon nanotubes: Tight-binding model," Physical Review B, vol. 75, no. 3, p. 035407, 2007. DOI:10.1103/PhysRevB.75.035407

[40] L. A. Agapito, M. Fornari, D. Ceresoli, A. Ferretti, S. Curtarolo, and M. B. Nardelli, "Accurate tight-binding Hamiltonians for twodimensional and layered materials," Physical Review B, vol. 93, no. 12, pp. 1-8, 2016. DOI:10.1103/PhysRevB.93.125137

[41] G. H. Wannier, "The structure of electronic excitation levels in insulating crystals," Physical Review, vol. 52, no. 3, p. 191 , 1937. DOI:10.1103/PhysRev.52.191

[42] N. Marzari, A. A. Mostofi, J. R. Yates, I. Souza, and D. Vanderbilt, "Maximally localized wannier functions: Theory and applications," Reviews of Modern Physics, vol. 84, no. 4, p. 1419, 2012. DOI:10.1103/RevModPhys.84.1419

[43] N. Marzari and D. Vanderbilt, "Maximally localized generalized Wannier functions for composite energy bands," Physical Review B Condensed Matter and Materials Physics, vol. 56, no. 20, pp. 12 847-12 865, 1997. DOI:10.1103/PhysRevB.56.12847

[44] I. Souza, N. Marzari, and D. Vanderbilt, "Maximally-localized Wannier functions for entangled energy bands," 2001. DOI:10.1103/PhysRevB.65.035109

[45] A. A. Mostofi, J. R. Yates, Y.-S. Lee, I. Souza, D. Vanderbilt, and N. Marzari, "wannier90: A tool for obtaining maximally-localised wannier functions," Computer physics communications, vol. 178, no. 9, pp. 685-699, 2008. DOI:10.1016/j.cpc.2007.11.016

[46] H. Zhang, C.-X. Liu, X.-L. Qi, X. Dai, Z. Fang, and S.-C. Zhang, "Topological insulators in Bi2Se3, Bi2Te3 and Sb2Te3 with a single Dirac cone on the surface," Nature Physics, vol. 5, no. 6, pp. 438-442, 2009. DOI:10.1038/nphys1270

[47] G. Pizzi, M. Gibertini, E. Dib, N. Marzari, G. Iannaccone, and G. Fiori, "Performance of arsenene and antimonene double-gate mosfets from first principles," Nature communications, vol. 7, p. 12585, 2016. DOI:10.1038/ncomms12585

[48] J. L. Lado and J. Fernández-Rossier, "Landau levels in 2D materials using Wannier Hamiltonians obtained by first principles," 2D Materials, vol. 3, no. 3, pp. 1-10, 2016. DOI:10.1088/2053-1583/3/3/035023

[49] E. G. Marin, D. Marian, G. Iannaccone, and G. Fiori, "First-principles simulations of fets based on two-dimensional inse," IEEE Electron Device Letters, 2018. DOI:10.1109/LED.2018.2804388

[50] Y. Ahn and M. Shin, "First-Principles-Based Quantum Transport Simulations of Monolayer Indium Selenide FETs in the Ballistic Limit," IEEE Transactions on Electron Devices, vol. 64, no. 5, pp. 2129-2134, 2017. DOI:10.1109/TED.2017.2679217

[51] A. K. A. Lu, G. Pourtois, M. Luisier, I. P. Radu, and M. Houssa, "On the electrostatic control achieved in transistors based on multilayered mos2: A first-principles study,” Journal of Applied Physics, vol. 121, no. 4, p. 044505, 2017. DOI:10.1063/1.4974960

[52] C. Stieger, A. Szabo, T. Bunjaku, and M. Luisier, "Ab-initio quantum transport simulation of self-heating in single-layer 2-d materials," Journal of Applied Physics, vol. 122, no. 4, p. 045708, 2017. DOI:10.1063/1.4990384

[53] Y.-S. Lee, M. B. Nardelli, and N. Marzari, "Band structure and quantum conductance of nanostructures from maximally localized wannier functions: The case of functionalized carbon nanotubes," Physical review letters, vol. 95, no. 7, p. $076804,2005$. DOI:10.1103/PhysRevLett.95.076804

[54] D. Marian, E. Dib, T. Cusati, E. G. Marin, A. Fortunelli, G. Iannaccone, and G. Fiori, "Transistor Concepts Based on Lateral Heterostructures of Metallic and Semiconducting Phases of MoS2," Physical Review Applied, vol. 8, no. 5, pp. 1-7, 2017. DOI:10.1103/PhysRevApplied.8.054047

[55] E. Marin, D. Marian, G. Iannaccone, and G. Fiori, "First principles investigation of tunnel fets based on nanoribbons from topological two-dimensional materials," Nanoscale, vol. 9, no. 48, pp. 19390-19397, 2017. DOI:10.1039/C7NR06015G

[56] A. Kormányos, G. Burkard, M. Gmitra, J. Fabian, V. Zólyomi, N. D. Drummond, and V. Fal'ko, "K.P Theory for Two-Dimensional Transition Metal Dichalcogenide Semiconductors," 2D Materials, 2014. DOI:10.1088/2053-1583/2/2/022001

[57] R. Landauer, "Spatial variation of currents and fields due to localized scatterers in metallic conduction," IBM Journal of Research and Development, vol. 1, no. 3, pp. 223-231, 1957.

[58] L. V. Keldysh et al., "Diagram technique for nonequilibrium processes," Sov. Phys. JETP, vol. 20, no. 4, pp. 1018-1026, 1965.

[59] S. Datta, "Nanoscale device modeling: the greens function method," Superlattices and microstructures, vol. 28, no. 4, pp. 253-278, 2000. DOI:10.1006/spmi.2000.0920

[60] M. B. Nardelli, "Electronic transport in extended systems: Application to carbon nanotubes," Physical Review B, vol. 60, no. 11, pp. 7828-7833, 1999. DOI:10.1103/PhysRevB.60.7828

[61] Y. Yoon, K. Ganapathi, and S. Salahuddin, "How good can monolayer mos2 transistors be?" Nano letters, vol. 11, no. 9, pp. 3768-3773, 2011. DOI:10.1021/n12018178 
[62] K. Gong, L. Zhang, D. Liu, L. Liu, Y. Zhu, Y. Zhao, and H. Guo, "Electric control of spin in monolayer wse2 field effect transistors," Nanotechnology, vol. 25, no. 43, p. 435201, 2014. DOI:10.1088/0957-4484/25/43/435201

[63] H. Lu and A. Seabaugh, "Tunnel field-effect transistors: State-of-the-art," IEEE Journal of the Electron Devices Society, vol. 2, no. 4, pp. 44-49, 2014. DOI:10.1109/JEDS.2014.2326622

[64] A. K. A. Lu, M. Houssa, M. Luisier, and G. Pourtois, "Impact of layer alignment on the behavior of mos 2- zrs 2 tunnel field-effect transistors: An ab initio study," Physical Review Applied, vol. 8, no. 3, p. 034017, 2017. DOI:10.1103/PhysRevApplied.8.034017

[65] A. Kuc, T. Cusati, E. Dib, A. F. Oliveira, A. Fortunelli, G. Iannaccone, T. Heine, and G. Fiori, "High-performance 2d p-type transistors based on gase layers: An ab initio study." DOI:10.1002/aelm.201600399

[66] D. Çakır, H. Sahin, and F. M. Peeters, "Tuning of the electronic and optical properties of single-layer black phosphorus by strain," Physical Review B, vol. 90, no. 20, p. 205421, 2014. DOI:10.1103/PhysRevB.90.205421

[67] F. Liu, Y. Wang, X. Liu, J. Wang, and H. Guo, "Ballistic transport in monolayer black phosphorus transistors," IEEE Transactions on Electron Devices, vol. 61, no. 11, pp. 3871-3876, 2014. DOI:10.1109/TED.2014.2353213

[68] M. S. Fahad, A. Srivastava, A. K. Sharma, C. Mayberry, and K. Mohsin, "Silicene nanoribbon tunnel field effect transistor," ECS Transactions, vol. 75, no. 5, pp. 175-181, 2016. DOI:10.1149/07505.0175ecst

[69] L. De Broglie, "An introduction to the study of wave mechanics," 1930.

[70] D. Bohm, "A suggested interpretation of the quantum theory in terms of" hidden" variables. i," Physical Review, vol. 85, no. 2, p. 166, 1952. DOI:10.1103/PhysRev.85.166

[71] D. Dürr, S. Goldstein, and N. Zanghì, Quantum physics without quantum philosophy. Springer Science \& Business Media, 2012.

[72] X. Oriols, "Quantum-trajectory approach to time-dependent transport in mesoscopic systems with electron-electron interactions," Physical review letters, vol. 98, no. 6, p. 066803, 2007. DOI:10.1103/PhysRevLett.98.066803

[73] E. Colomés, Z. Zhan, D. Marian, and X. Oriols, "Quantum dissipation with conditional wave functions: Application to the realistic simulation of nanoscale electron devices," Physical Review B, vol. 96, no. 7, p. 075135, 2017. DOI:10.1103/PhysRevB.96.075135

[74] J. David, L. Register, and S. Banerjee, "Semiclassical monte carlo analysis of graphene fets," IEEE Transactions on Electron Devices, vol. 59, no. 4, pp. 976-982, 2012. DOI:10.1109/TED.2012.2184116

[75] P. Buividovich and M. Polikarpov, "Monte carlo study of the electron transport properties of monolayer graphene within the tight-binding moedl." DOI:10.1103/PhysRevB.86.245117

[76] T. Fang, A. Konar, H. Xing, and D. Jena, "High-field transport in two-dimensional graphene," Physical Review B, vol. 84, no. 12, p. 125450, 2011. DOI:10.1103/PhysRevB.84.125450

[77] D. Ferry, "Electron transport in some transition metal di-chalcogenides: Mos2 and ws2," Semiconductor Science and Technology, vol. 32, no. 8, p. 085003, 2017. DOI:10.1088/1361-6641/aa7472

[78] G. Gaddemane, W. G. Vandenberghe, and M. V. Fischetti, "Theoretical study of electron transport in silicene and germanene using full-band monte carlo simulations," in (SISPAD), 2016. IEEE, 2016, pp. 353-356.

[79] D. Esseni, P. Palestri, and L. Selmi, Nanoscale MOS transistors: semi-classical transport and applications. Cambridge University Press, 2011.

[80] R. Kubo, "A general expression for the conductivity tensor," Canadian Journal of Physics, vol. 34, no. 12A, pp. 1274-1277, 1956. DOI:10.1139/p56-140

[81] D. Greenwood, "The boltzmann equation in the theory of electrical conduction in metals," Proceedings of the Physical Society, vol. 71, no. 4, p. 585, 1958. DOI:10.1088/0370-1328/71/4/306

[82] T. Fang, A. Konar, H. Xing, and D. Jena, "Mobility in semiconducting graphene nanoribbons: Phonon, impurity, and edge roughness scattering," Physical Review B, vol. 78, no. 20, p. 205403, 2008. DOI:10.1103/PhysRevB.78.205403

[83] A. Konar, T. Fang, and D. Jena, "Effect of high- $\kappa$ gate dielectrics on charge transport in graphene-based field effect transistors," Physical Review B, vol. 82, no. 11, p. 115452, 2010. DOI:10.1103/PhysRevB.82.115452

[84] A. Betti, G. Fiori, and G. Iannaccone, "Atomistic investigation of low-field mobility in graphene nanoribbons," IEEE Transactions on Electron Devices, vol. 58, no. 9, pp. 2824-2830, 2011. DOI:10.1109/TED.2010.2100045

[85] Z.-Y. Ong and M. V. Fischetti, "Mobility enhancement and temperature dependence in top-gated single-layer mos 2," Physical Review B, vol. 88, no. 16, p. 165316, 2013. DOI:10.1103/PhysRevB.88.165316

[86] B. Liao, J. Zhou, B. Qiu, M. S. Dresselhaus, and G. Chen, "Ab initio study of electron-phonon interaction in phosphorene," Physical Review B, vol. 91, no. 23, p. 235419, 2015. DOI:10.1103/PhysRevB.91.235419

[87] M. V. Fischetti and W. G. Vandenberghe, "Mermin-wagner theorem, flexural modes, and degraded carrier mobility in two-dimensional crystals with broken horizontal mirror symmetry," Physical Review B, vol. 93, no. 15, p. 155413, 2016. DOI:10.1103/PhysRevB.93.155413

[88] D. Vasileska and S. M. Goodnick, Computational Electronics : Semiclassical and quantum device modeling and simulation, 2006, vol. 1, no. 1.

[89] W. Hänsch, The drift diffusion equation and its applications in MOSFET modeling. Springer Science \& Business Media, 2012.

[90] M. G. Ancona, "Electron transport in graphene from a diffusion-drift perspective," IEEE Transactions on Electron Devices, vol. 57, no. 3, pp. 681-689, 2010. DOI:10.1109/TED.2009.2038644

[91] A. Paussa, M. Geromel, P. Palestri, M. Bresciani, D. Esseni, and L. Selmi, "Simulation of graphene nanoscale rf transistors including scattering and generation/recombination mechanisms," in Electron Devices Meeting (IEDM), 2011 IEEE International. IEEE, 2011, pp. $11-7$.

[92] A. Y. Serov, Z. Y. Ong, M. V. Fischetti, and E. Pop, “Theoretical analysis of high-field transport in graphene on a substrate," Journal of Applied Physics, vol. 116, no. 3, 2014. DOI:10.1063/1.4884614

[93] R. Bistritzer and A. MacDonald, "Hydrodynamic theory of transport in doped graphene," Physical Review B, vol. 80, no. 8, p. 085109, 2009. DOI:10.1103/PhysRevB.80.085109

[94] S. Kaneko, H. Tsuchiya, Y. Kamakura, N. Mori, and M. Ogawa, "Theoretical performance estimation of silicene, germanene, and graphene nanoribbon field-effect transistors under ballistic transport," Applied Physics Express, vol. 7, no. 3, pp. 3-7, 2014. DOI:10.7567/APEX.7.035102 
[95] E. G. Marin, S. J. Bader, and D. Jena, "A new holistic model of 2-d semiconductor fets," IEEE Transactions on Electron Devices, vol. 65, no. 3, pp. 1239-1245, 2018. DOI:10.1109/TED.2018.2797172

[96] S. V. Suryavanshi and E. Pop, "S2ds: Physics-based compact model for circuit simulation of two-dimensional semiconductor devices including non-idealities," Journal of Applied Physics, vol. 120, no. 22, p. 224503, 2016. DOI:10.1063/1.4971404

[97] W. Cao, J. Kang, W. Liu, and K. Banerjee, "A compact current-voltage model for 2d semiconductor based field-effect transistors considering interface traps, mobility degradation, and inefficient doping effect," IEEE Transactions on Electron Devices, vol. 61, no. 12, pp. 4282-4290, 2014. DOI:10.1109/TED.2014.2365028

[98] D. Jiménez, "Drift-diffusion model for single layer transition metal dichalcogenide field-effect transistors," Applied Physics Letters, vol. 101, no. 24, p. 243501, 2012. DOI:10.1063/1.4770313

[99] S.-J. Liang and L. Ang, "Electron thermionic emission from graphene and a thermionic energy converter," Physical Review Applied, vol. 3, no. 1, p. 014002, 2015. DOI:10.1103/PhysRevApplied.3.014002

[100] X. Wei, Q. Chen, and L. Peng, "Electron emission from a two-dimensional crystal with atomic thickness," AIP Advances, vol. 3, no. 4, p. 042130, 2013. DOI:10.1063/1.4802973

[101] D. Jena, "Tunneling transistors based on graphene and 2-D Crystals," Proceedings of the IEEE, vol. 101, no. 7, pp. 1585-1602, 2013. DOI:10.1109/JPROC.2013.2253435

[102] R. M. Feenstra, D. Jena, and G. Gu, "Single-particle tunneling in doped graphene-insulator-graphene junctions," Journal of Applied Physics, vol. 111, no. 4, p. 043711, 2012. DOI:10.1063/1.3686639

[103] "International Technology Roadmap for Semiconductors: http://www.itrs2.net/."

[104] "IEEE International Roadmap for Devices and Systems: http://irds.ieee.org/."

[105] A. C. Ferrari, F. Bonaccorso, V. Fal'ko, K. S. Novoselov, S. Roche, P. Bøggild, S. Borini, F. H. L. Koppens, and e. a. Palermo, "Science and technology roadmap for graphene, related two-dimensional crystals, and hybrid systems," Nanoscale, vol. 7, no. 11, pp. 4598-4810, 2015. DOI:10.1039/C4NR01600A

[106] F. Schwierz, "Graphene transistors," Nature Nanotechnology, vol. 5, no. 7, pp. 487-496, 2010. DOI:10.1038/nnano.2010.89

[107] P. Zhao, R. M. Feenstra, G. Gu, and D. Jena, "Symfet: A proposed symmetric graphene tunneling field-effect transistor," IEEE Transactions on Electron Devices, vol. 60, no. 3, pp. 951-957, 2013. DOI:10.1109/TED.2013.2238238

[108] M. Cheli, G. Fiori, and G. Iannaccone, "A semianalytical model of bilayer-graphene field-effect transistor," IEEE Transactions on Electron Devices, vol. 56, no. 12, pp. 2979-2986, 2009. DOI:10.1109/TED.2009.2033419

[109] G. Fiori and G. Iannaccone, "Ultralow-voltage bilayer graphene tunnel fet," IEEE Electron Device Letters, vol. 30, no. 10, pp. 1096-1098, 2009. DOI:10.1109/LED.2009.2028248

[110] P. Zhao, J. Chauhan, and J. Guo, "Computational Study of Tunneling Transistor Based on Graphene Nanoribbon," Nano Letters, vol. 9, no. 2, pp. 684-688, 2009. DOI:10.1021/n1803176x

[111] A. Molle, J. Goldberger, M. Houssa, Y. Xu, S. C. Zhang, and D. Akinwande, "Buckled two-dimensional Xene sheets," Nature Materials, vol. 16, no. 2, pp. 163-169, 2017. DOI:10.1038/nmat4802

[112] M. Houssa, A. Dimoulas, and A. Molle, "Silicene: A review of recent experimental and theoretical investigations," Journal of Physics Condensed Matter, vol. 27, no. 25, 2015. DOI:10.1088/0953-8984/27/25/253002

[113] L. Matthes and F. Bechstedt, "Influence of edge and field effects on topological states of germanene nanoribbons from self-consistent calculations," Physical Review B - Condensed Matter and Materials Physics, vol. 90, no. 16, pp. 1-7, 2014. DOI:10.1103/PhysRevB.90.165431

[114] Z. Ni, Q. Liu, K. Tang, J. Zheng, J. Zhou, R. Qin, Z. Gao, D. Yu, and J. Lu, “Tunable bandgap in silicene and germanene,” Nano Letters, vol. 12, no. 1, pp. 113-118, 2012. DOI:10.1021/nl203065e

[115] Y. Xu, B. Yan, H. J. Zhang, J. Wang, G. Xu, P. Tang, W. Duan, and S. C. Zhang, "Large-gap quantum spin hall insulators in tin films," Physical Review Letters, vol. 111, no. 13, pp. 1-5, 2013. DOI:10.1103/PhysRevLett.111.136804

[116] M. Houssa, B. van den Broek, K. Iordanidou, A. K. A. Lu, G. Pourtois, J. P. Locquet, V. Afanas'ev, and A. Stesmans, “Topological to trivial insulating phase transition in stanene," Nano Research, vol. 9, no. 3, pp. 774-778, 2016. DOI:10.1007/s12274-015-0956-y

[117] M. Ezawa, "Topological electronics and topological field effect transistor in silicene, germanene and stanene," IEEE-NANO 2015 - 15th International Conference on Nanotechnology, no. 4, pp. 604-608, 2015. DOI:10.1109/NANO.2015.7388677

[118] N. B. Le, T. D. Huan, and L. M. Woods, "Tunable Spin-Dependent Properties of Zigzag Silicene Nanoribbons," Physical Review Applied, vol. 1, no. 5, pp. 1-6, 2014. DOI:10.1103/PhysRevApplied.1.054002

[119] A. Hattori, S. Tanaya, K. Yada, M. Araidai, M. Sato, Y. Hatsugai, K. Shiraishi, and Y. Tanaka, "Edge states of hydrogen terminated monolayer materials: Silicene, germanene and stanene ribbons," Journal of Physics Condensed Matter, vol. 29, no. 11, pp. 1-12, 2017. DOI:10.1088/1361-648X/aa57e0

[120] W. G. Vandenberghe and M. V. Fischetti, "Imperfect two-dimensional topological insulator field-effect transistors," Nature Communications, vol. 8, pp. 1-8, 2017. DOI:10.1038/ncomms14184

[121] B. van den Broek, M. Houssa, G. Pourtois, V. V. Afanas'ev, and A. Stesmans, "Current-voltage characteristics of armchair Sn nanoribbons," Physica Status Solidi - Rapid Research Letters, vol. 8, no. 11, pp. 931-934, 2014. DOI:10.1002/pssr.201400073

[122] Y. Ding, Y. Wang, J. Ni, L. Shi, S. Shi, and W. Tang, "First principles study of structural, vibrational and electronic properties of graphene-like MX2(M=Mo, Nb, W, Ta; X=S, Se, Te) monolayers," Physica B: Condensed Matter, vol. 406, no. 11, pp. 2254-2260, 2011. DOI:10.1016/j.physb.2011.03.044

[123] T. Heine, "Transition metal chalcogenides: Ultrathin inorganic materials with tunable electronic properties," Accounts of Chemical Research, vol. 48, no. 1, pp. 65-72, 2015. DOI:10.1021/ar500277z

[124] G.-B. Liu, D. Xiao, Y. Yao, X. Xu, and W. Yao, "Electronic structures and theoretical modelling of two-dimensional group-VIB transition metal dichalcogenides," Chem. Soc. Rev., vol. 44, no. 9, pp. 2643-2663, 2015. DOI:10.1039/C4CS00301B

[125] M. Shahriari, A. G. Dezfuli, and M. Sabaeian, "Band structure and orbital character of monolayer mos 2 with eleven-band tight-binding model," Superlattices and Microstructures, 2017. DOI:10.1016/j.spmi.2017.12.030

[126] D. Xiao, G.-B. Liu, W. Feng, X. Xu, and W. Yao, "Coupled spin and valley physics in monolayers of mos 2 and other group-vi dichalcogenides," Physical Review Letters, vol. 108, no. 19, p. 196802, 2012. DOI:10.1103/PhysRevLett.108.196802 
[127] T. Agarwal, D. Yakimets, P. Raghavan, I. Radu, A. Thean, S. Member, M. Heyns, W. Dehaene, and S. Member, "Benchmarking of MoS 2 FETs With Multigate Si-FET Options for 5 nm and Beyond," vol. 62, no. 12, pp. 4051-4056, 2015. DOI:10.1109/TED.2015.2491021

[128] Y. Li, K. A. N. Duerloo, K. Wauson, and E. J. Reed, "Structural semiconductor-to-semimetal phase transition in two-dimensional materials induced by electrostatic gating," Nature Communications, vol. 7, pp. 1-8, 2016. DOI:10.1038/ncomms 10671

[129] Y. C. Lin, D. O. Dumcenco, Y. S. Huang, and K. Suenaga, "Atomic mechanism of the semiconducting-to-metallic phase transition in single-layered MoS 2," Nature Nanotechnology, vol. 9, no. 5, pp. 391-396, 2014. DOI:10.1038/nnano.2014.64

[130] Y. Wang, J. Xiao, H. Zhu, Y. Li, Y. Alsaid, K. Y. Fong, Y. Zhou, S. Wang, W. Shi, Y. Wang, A. Zettl, E. J. Reed, and X. Zhang, "Structural phase transition in monolayer MoTe2 driven by electrostatic doping," Nature, vol. 550, no. 7677, pp. 487-491, 2017. DOI:10.1038/nature24043

[131] P. Mirõ, M. Ghorbani-Asl, and T. Heine, "Two dimensional materials beyond MoS2: Noble-transition-metal dichalcogenides," Angewandte Chemie - International Edition, vol. 53, no. 11, pp. 3015-3018, 2014. DOI:10.1002/anie.201309280

[132] J. Sun, H. Shi, T. Siegrist, D. J. Singh, J. Sun, H. Shi, T. Siegrist, and D. J. Singh, "Electronic , transport , and optical properties of bulk and mono-layer PdSe2 Electronic, transport, and optical properties of bulk and mono-layer PdSe 2," vol. 153902, no. 2015, pp. 2-6, 2015. DOI:10.1063/1.4933302

[133] W. Zhang, Z. Huang, W. Zhang, and Y. Li, "Two-dimensinonal semiconductors with possible high room temperature mobility," Nano Research, vol. 7, no. 12, pp. 1731-1737, 2014.

[134] M. Ghorbani-Asl, A. Kuc, P. Miró, and T. Heine, “A Single-Material Logical Junction Based on 2D Crystal PdS2,” Advanced Materials, vol. 28, no. 5, pp. 853-856, 2016. DOI:10.1002/adma.201504274

[135] A. Jain and A. J. McGaughey, "Strongly anisotropic in-plane thermal transport in single-layer black phosphorene," Scientific Reports, vol. 5, pp. 1-5, 2015. DOI:10.1038/srep08501

[136] F. Xia, H. Wang, and Y. Jia, "Rediscovering black phosphorus as an anisotropic layered material for optoelectronics and electronics," Nature Communications, vol. 5, pp. 1-6, 2014. DOI:10.1038/ncomms5458

[137] L. Li, Y. Yu, G. J. Ye, Q. Ge, X. Ou, H. Wu, D. Feng, X. H. Chen, and Y. Zhang, "Black phosphorus field-effect transistors," Nature Nanotechnology, vol. 9, no. 5, pp. 372-377, 2014. DOI:10.1038/nnano.2014.35

[138] Q. Wu, L. Shen, M. Yang, Y. Cai, Z. Huang, and Y. P. Feng, "Electronic and transport properties of phosphorene nanoribbons," Physical Review B - Condensed Matter and Materials Physics, vol. 92, no. 3, pp. 1-9, 2015. DOI:10.1103/PhysRevB.92.035436

[139] J. Chang and C. Hobbs, "Theoretical study of phosphorene tunneling field effect transistors," Applied Physics Letters, vol. 106, no. 8, 2015. DOI: $10.1063 / 1.4913842$

[140] H. Guo, N. Lu, J. Dai, X. Wu, and X. C. Zeng, "Phosphorene nanoribbons, phosphorus nanotubes, and van der Waals multilayers," Journal of Physical Chemistry C, vol. 118, no. 25, pp. 14051-14059, 2014. DOI:10.1021/jp505257g

[141] Z. Zhu and D. Tománek, "Semiconducting layered blue phosphorus: A computational study," Physical Review Letters, vol. 112, no. 17, pp. 1-5, 2014. DOI:10.1103/PhysRevLett.112.176802

[142] S. Zhang, M. Xie, F. Li, Z. Yan, Y. Li, E. Kan, W. Liu, Z. Chen, and H. Zeng, "Semiconducting Group 15 Monolayers: A Broad Range of Band Gaps and High Carrier Mobilities," Angewandte Chemie - International Edition, vol. 55, no. 5, pp. 1666-1669, 2016. DOI:10.1002/anie.201507568

[143] Y. Wang and Y. Ding, "Electronic Structure and Carrier Mobilities of Arsenene and Antimonene Nanoribbons: A First-Principle Study," Nanoscale Research Letters, vol. 10, no. 1, pp. 1-10, 2015. DOI:10.1186/s11671-015-0955-7

[144] E. Aktürk, O. Ü. Aktürk, and S. Ciraci, "Single and bilayer bismuthene: Stability at high temperature and mechanical and electronic properties," Physical Review B, vol. 94, no. 1, pp. 1-9, 2016. DOI:10.1103/PhysRevB.94.014115

[145] F. Reis, G. Li, L. Dudy, M. Bauernfeind, S. Glass, W. Hanke, R. Thomale, J. Schäfer, and R. Claessen, "Bismuthene on a SiC substrate: A candidate for a high-temperature quantum spin Hall material," Science, vol. 357, no. 6348, pp. 287-290, 2017. DOI:10.1126/science.aai8142

[146] Q. Zhang, G. Iannaccone, and G. Fiori, “Two-Dimensional Tunnel Transistors Based on Bi2Se3 Thin Film," IEEE Electron Device Letters, vol. 35, no. 1, pp. 129-131, 2014. DOI:10.1109/LED.2013.2288036

[147] S. Zhang, N. Wang, S. Liu, S. Huang, W. Zhou, B. Cai, M. Xie, Q. Yang, X. Chen, and H. Zeng, "Two-dimensional GeS with tunable electronic properties via external electric field and strain," Nanotechnology, vol. 27, no. 27, 2016. DOI:10.1088/0957-4484/27/27/274001

[148] Z. Q. Fan, X. W. Jiang, Z. Wei, J. W. Luo, and S. S. Li, "Tunable Electronic Structures of GeSe Nanosheets and Nanoribbons," Journal of Physical Chemistry C, vol. 121, no. 26, pp. 14373-14379, 2017. DOI:10.1021/acs.jpcc.7b04607

[149] R. Fei, W. Kang, and L. Yang, "Ferroelectricity and phase transitions in monolayer group-iv monochalcogenides," Physical review letters, vol. 117, no. 9, p. 097601, 2016. DOI:10.1103/PhysRevLett.117.097601

[150] L. Debbichi, O. Eriksson, and S. Lebègue, "Two-Dimensional Indium Selenides Compounds: An Ab Initio Study," Journal of Physical Chemistry Letters, vol. 6, no. 15, pp. 3098-3103, 2015. DOI:10.1021/acs.jpclett.5b01356

[151] L. Huang, Z. Chen, and J. Li, "Effects of strain on the band gap and effective mass in two-dimensional monolayer GaX (X= S, Se, Te)," RSC Adv., vol. 5, no. 8, pp. 5788-5794, 2015. DOI:10.1039/C4RA12107D

[152] Y. Ma, Y. Dai, L. Yu, C. Niu, and B. Huang, "Engineering a topological phase transition in $\beta$-InSe via strain," New Journal of Physics, vol. 15, 2013. DOI:10.1088/1367-2630/15/7/073008

[153] D. Saha and S. Mahapatra, "Atomistic modeling of the metallic-to-semiconducting phase boundaries in monolayer MoS2," vol. 253106, pp. 1-5, 2016. DOI:10.1063/1.4954257

[154] R. Hafizi, J. Tersoff, and V. Perebeinos, "Band Structure and Contact Resistance of Carbon Nanotubes Deformed by a Metal Contact," Physical Review Letters, vol. 119, no. 20, pp. 1-5, 2017. DOI:10.1103/PhysRevLett.119.207701

[155] G. Giovannetti, P. Khomyakov, G. Brocks, V. v. Karpan, J. Van den Brink, and P. J. Kelly, "Doping graphene with metal contacts," Physical review letters, vol. 101, no. 2, p. 026803, 2008. DOI:10.1103/PhysRevLett.101.026803

[156] T. Cusati, G. Fiori, A. Gahoi, V. Passi, M. C. Lemme, A. Fortunelli, and G. Iannaccone, "Electrical properties of graphene-metal contacts," Scientific Reports, vol. 7, no. 1, p. 5109, 2017. DOI:10.1038/s41598-017-05069-7

[157] D. Jariwala, T. J. Marks, and M. C. Hersam, "Mixed-dimensional van der Waals heterostructures," Nature Materials, vol. 16, no. 2, pp. 170-181, 2017. DOI:10.1038/nmat4703 
[158] C. Guo, T. Wang, C. Xia, and Y. Liu, "Modulation of electronic transport properties in armchair phosphorene nanoribbons by doping and edge passivation," Scientific Reports, vol. 7, no. 1, pp. 1-8, 2017. DOI:10.1038/s41598-017-13212-7

[159] N. K. Jaiswal, N. Tyagi, A. Kumar, and P. Srivastava, "Inducing half-metallicity with enhanced stability in zigzag graphene nanoribbons via fluorine passivation," Applied Surface Science, vol. 396, pp. 471-479, 2017. DOI:10.1016/j.apsusc.2016.10.178

[160] M. Sagynbaeva, P. Panigrahi, L. Yunguo, M. Ramzan, and R. Ahuja, "Tweaking the magnetism of mos2 nanoribbon with hydrogen and carbon passivation," Nanotechnology, vol. 25, no. 16, p. 165703, 2014. DOI:10.1088/0957-4484/25/16/165703

[161] D. Wang, L. Chen, C. Shi, X. Wang, G. Cui, P. Zhang, and Y. Chen, "Quantum spin Hall insulator in halogenated arsenene films with sizable energy gaps,” Scientific Reports, vol. 6, no. June, pp. 1-7, 2016. DOI:10.1038/srep28487

[162] S. Radhakrishnan, D. Das, A. Samanta, C. A. de los Reyes, L. Deng, L. B. Alemany, T. K. Weldeghiorghis, V. N. Khabashesku, V. Kochat, Z. Jin, P. M. Sudeep, A. A. Martí, C.-W. Chu, A. Roy, C. S. Tiwary, A. K. Singh, and P. M. Ajayan, "Fluorinated h-BN as a magnetic semiconductor," Science Advances, vol. 3, no. 7, p. e1700842, 2017. DOI:10.1126/sciadv.1700842

[163] K. S. Novoselov, A. Mishchenko, A. Carvalho, and A. H. Castro Neto, "2D materials and van der Waals heterostructures," Science, vol. 353, no. 6298, 2016. DOI:10.1126/science.aac9439

[164] A. P. Rooney, A. Kozikov, A. N. Rudenko, E. Prestat, M. J. Hamer, F. Withers, Y. Cao, K. S. Novoselov, M. I. Katsnelson, R. Gorbachev, and S. J. Haigh, "Observing Imperfection in Atomic Interfaces for van der Waals Heterostructures," Nano Letters, vol. 17, no. 9, pp. 5222-5228, 2017. DOI:10.1021/acs.nanolett.7b01248

[165] F. Chen, H. Ilatikhameneh, Y. Tan, D. Valencia, G. Klimeck, and R. Rahman, "Transport in vertically stacked hetero-structures from 2D materials," Journal of Physics: Conference Series, vol. 864, no. 1, pp. 8-12, 2017. DOI:10.1088/1742-6596/864/1/012053

[166] X. Qian, Y. Wang, W. Li, J. Lu, and J. Li, "Modelling of stacked 2D materials and devices," 2D Materials, vol. 2, no. 3, 2015. DOI:10.1088/2053-1583/2/3/032003

[167] S. Bruzzone, D. Logoteta, G. Fiori, and G. Iannaccone, "Vertical transport in graphene-hexagonal boron nitride heterostructure devices," Scientific Reports, vol. 5, no. Section II, pp. 1-10, 2015. DOI:10.1038/srep14519

[168] G. Iannaccone, Q. Zhang, S. Bruzzone, and G. Fiori, "Insights on the physics and application of off-plane quantum transport through graphene and 2D materials," Solid-State Electronics, vol. 115, pp. 213-218, 2016. DOI:10.1016/j.sse.2015.08.008

[169] D. McManus, S. Vranic, F. Withers, V. Sanchez-Romaguera, M. Macucci, H. Yang, R. Sorrentino, K. Parvez, S. K. Son, G. Iannaccone, K. Kostarelos, G. Fiori, and C. Casiraghi, "Water-based and biocompatible 2D crystal inks for all-inkjet-printed heterostructures," Nature Nanotechnology, vol. 12, no. 4, pp. 343-350, 2017. DOI:10.1038/nnano.2016.281

[170] S. Kumar, J. Y. Murthy, and M. A. Alam, "Percolating conduction in finite nanotube networks," Physical Review Letters, vol. 95, no. 6, pp. 3-6, 2005. DOI:10.1103/PhysRevLett.95.066802

[171] S. Colasanti, G. Cheng, P. der Dissertation, P. Lugli, and S. Goodnick, "Modeling and Simulation of Carbon Nanotubes Networks: Toward a Multiscale Approach," 2016.

[172] S. P. Schieß1, X. de Vries, M. Rother, A. Massé, M. Brohmann, P. A. Bobbert, and J. Zaumseil, "Modeling carrier density dependent charge transport in semiconducting carbon nanotube networks," Physical Review Materials, vol. 1, no. 4, p. $046003,2017$. DOI:10.1103/PhysRevMaterials.1.046003 